\title{
K2 Observations of SN 2018oh Reveal a Two-component Rising Light Curve for a Type Ia Supernova
}

G. Dimitriadis ${ }^{1}$, R. J. Foley ${ }^{1}$, A. Rest ${ }^{2,3}$, D. Kasen ${ }^{4,5}$, A. L. Piro ${ }^{6}$ (), A. Polin ${ }^{4,7}$, D. O. Jones ${ }^{1}$ (1),

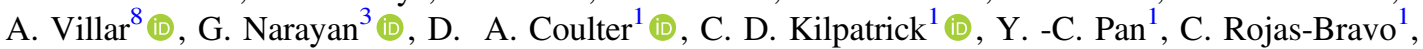
O. D. Fox ${ }^{2}$ (1) , S. W. Jha ${ }^{9}$ (1) P. E. Nugent ${ }^{4,7}$ (1) A. G. Riess ${ }^{2,3}$, D. Scolnic ${ }^{10}$, M. R. Drout ${ }^{6,62}$

K2 Mission Team,

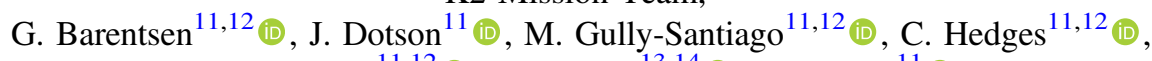

A. M. Cody ${ }^{11,12}$ (DD, T. Barclay ${ }^{13,14}$ (D), S. Howell ${ }^{11}$ (D)

KEGS,

P. Garnavich ${ }^{15}$ (iD , B. E. Tucker ${ }^{16,17,18}$ (D) E. Shaya ${ }^{19}$ (D), R. Mushotzky ${ }^{19}$, R. P. Olling ${ }^{19}$, S. Margheim ${ }^{20}$ (iD) A. Zenteno $^{21}$

Kepler spacecraft team,

J. Coughlin, J. E. Van Cleve ${ }^{11,22}$, J. Vinícius de Miranda Cardoso ${ }^{11,23}$, K. A. Larson ${ }^{24}$, K. M. McCalmont-Everton ${ }^{24}$, C. A. Peterson ${ }^{24}$, S. E. Ross ${ }^{24}$, L. H. Reedy ${ }^{25}$, D. Osborne ${ }^{25}$, C. McGinn ${ }^{25}$, L. Kohnert ${ }^{25}$, L. Migliorini ${ }^{25}$, A. Wheaton ${ }^{25}$, B. Spencer ${ }^{25}$, C. Labonde ${ }^{25}$, G. Castillo ${ }^{25}$, G. Beerman ${ }^{25}$, K. Steward ${ }^{25}$, M. Hanley ${ }^{25}$, R. Larsen ${ }^{25}$, R. Gangopadhyay ${ }^{25}$, R. Kloetzel $^{25}$, T. Weschler ${ }^{25}$, V. Nystrom ${ }^{25}$, J. Moffatt ${ }^{25}$, M. Redick ${ }^{25}$, K. Griest ${ }^{25}$, M. Packard ${ }^{25}$, M. Muszynski ${ }^{25}$, J. Kampmeier ${ }^{25}$, R. Bjella ${ }^{25}$, S. Flynn ${ }^{25}$, B. Elsaesser ${ }^{25}$ Pan-STARRS,

K. C. Chambers ${ }^{26}$ (D) H. A. Flewelling ${ }^{26}$ (D) M. E. Huber $^{26}$ (D) E. A. Magnier ${ }^{26}$, C. Z. Waters ${ }^{26}$, A. S. B. Schultz ${ }^{26}$, J. Bulger ${ }^{26}$, T. B. Lowe ${ }^{26}$, M. Willman ${ }^{26}$, S. J. Smartt ${ }^{27}$ (iD) K. W. Smith ${ }^{27}$

DECam,

S. Points ${ }^{21}$, G. M. Strampelli ${ }^{2,28}$

ASAS-SN,

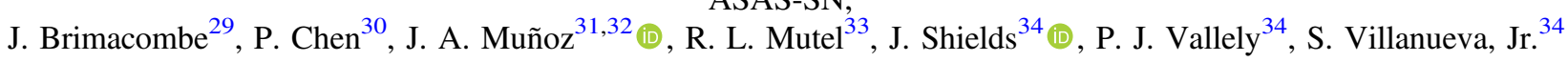
PTSS/TNTS,

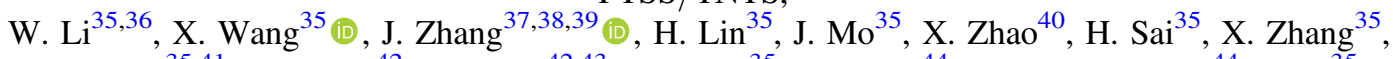

K. Zhang ${ }^{35,41}$, T. Zhang ${ }^{42}$, L. Wang ${ }^{42,43}$, J. Zhang ${ }^{35}$, E. Baron ${ }^{44}$ (iD, J. M. DerKacy ${ }^{44}$, L. Li ${ }^{35}$,

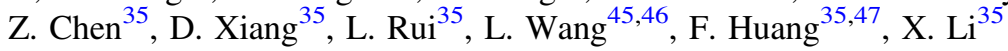

Las Cumbres Observatory,

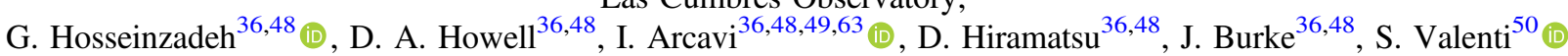

ATLAS,

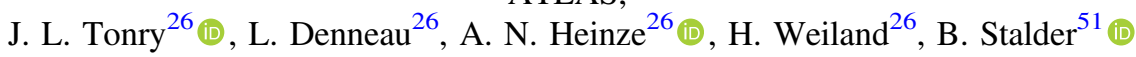

Konkoly,

J. Vinkó ${ }^{41,52,53}$ (D), K. Sárneczky ${ }^{52}$ (D) A. Pál ${ }^{52}$ (D), A. Bódi ${ }^{52,54}$, Zs. Bognár ${ }^{52}$ (D) B. Csák ${ }^{52}$, B. Cseh ${ }^{52}$, G. Csörnyei ${ }^{52}$,

O. Hanyecz ${ }^{52}$ (D) B. Ignácz ${ }^{52}$, Cs. Kalup ${ }^{52}$, R. Könyves-Tóth ${ }^{52}$, L. Kriskovics ${ }^{52}$, A. Ordasi ${ }^{52}$, I. Rajmon ${ }^{55}$, A. Sódor ${ }^{52}$, R. Szabó ${ }^{52}$ (iD), R. Szakáts ${ }^{52}$ (D), G. Zsidi ${ }^{52}$

ePESSTO,

and

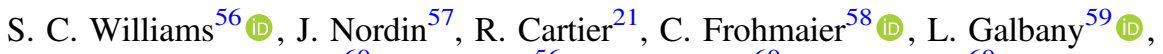

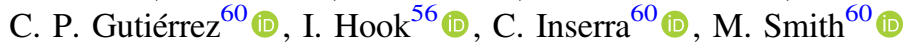

University of Arizona,

D. J. Sand ${ }^{61}$ (D) J. E. Andrews ${ }^{61}$, N. Smith ${ }^{61}$, and C. Bilinski ${ }^{61}$

${ }^{1}$ Department of Astronomy and Astrophysics, University of California, Santa Cruz, CA 95064, USA

${ }^{2}$ Space Telescope Science Institute, 3700 San Martin Drive, Baltimore, MD 21218, USA

${ }^{3}$ Department of Physics and Astronomy, Johns Hopkins University, Baltimore, MD 21218, USA

${ }^{4}$ Department of Astronomy, University of California, Berkeley, CA 94720-3411, USA

${ }^{5}$ Lawrence Berkeley National Laboratory, 1 Cyclotron Road, Berkeley, California 94720, USA

6 The Observatories of the Carnegie Institution for Science, 813 Santa Barbara Street, Pasadena, CA 91101, USA

${ }^{7}$ Lawrence Berkeley National Laboratory, Berkeley, CA, 94720, USA

${ }^{8}$ Harvard-Smithsonian Center for Astrophysics, 60 Garden Street, Cambridge, MA 02138, USA

${ }^{9}$ Department of Physics and Astronomy, Rutgers, The State University of New Jersey, 136 Frelinghuysen Road, Piscataway, NJ 08854, USA

${ }^{10}$ Kavli Institute for Cosmological Physics, University of Chicago, Chicago, IL 60637, USA

${ }^{11}$ NASA Ames Research Center, Moffett Field, CA 94035, USA

${ }_{13}^{12}$ Bay Area Environmental Research Institute, P.O. Box 25, Moffett Field, CA 94035, USA

${ }_{14}^{13}$ NASA Goddard Space Flight Center, 8800 Greenbelt Road, Greenbelt, MD 20771, USA

${ }^{14}$ University of Maryland, Baltimore County, 1000 Hilltop Circle, Baltimore, MD 21250, USA

${ }^{15}$ Department of Physics, University of Notre Dame, 225 Nieuwland Science Hall, Notre Dame, IN 46556-5670, USA

${ }^{16}$ The Research School of Astronomy and Astrophysics, Mount Stromlo Observatory, Australian National University, Canberra ACT 2611, Australia

${ }^{17}$ National Centre for the Public Awareness of Science, Australian National University, Canberra ACT 2611, Australia

${ }^{18}$ The ARC Centre of Excellence for All-Sky Astrophysics in 3 Dimensions (ASTRO 3D), Australia 


$$
\begin{aligned}
& { }^{19} \text { Astronomy Department, University of Maryland, College Park, MD 20742-2421, USA } \\
& { }_{20} \text { Gemini Observatory, La Serena, Chile } \\
& { }^{21} \text { Cerro Tololo Inter-American Observatory, Casilla 603, La Serena, Chile } \\
& { }^{22} \text { SETI Institute, } 189 \text { Bernardo Avenue, Mountain View, CA 94043, USA } \\
& 23 \text { Universidade Federal de Campina Grande, Campina Grande, Brazil } \\
& { }^{24} \text { Ball Aerospace and Technologies Corp., Boulder, CO 80301, USA } \\
& { }^{25} \text { LASP, University of Colorado at Boulder, Boulder, CO 80303, USA } \\
& { }^{26} \text { Institute of Astronomy, University of Hawaii, 2680 Wood- lawn Drive, Honolulu, HI 96822, USA } \\
& { }^{27} \text { Astrophysics Research Centre, School of Mathematics and Physics, Queens University Belfast, Belfast BT7 1NN, UK } \\
& \text { University of La Laguna, Calle Padre Herrera, E-38200 San Cristóbal de La Laguna, Santa Cruz de Tenerife, Spain }
\end{aligned}
$$

${ }^{29}$ Coral Towers Observatory, Cairns, Queensland 4870, Australia

${ }^{30}$ Kavli Institute for Astronomy and Astrophysics, Peking University, Yi He Yuan Road 5, Hai Dian District, Beijing 100871, People's Republic of China

${ }^{31}$ Departamento de Astronomía y Astrofísica, Universidad de Valencia, E-46100 Burjassot, Valencia, Spain

${ }_{32}$ Observatorio Astronómico, Universidad de Valencia, E-46980 Paterna, Valencia, Spain

${ }^{33}$ Department of Physics and Astronomy, University of Iowa, Iowa City, IA 52242, USA

${ }^{34}$ Department of Astronomy, The Ohio State University, 140 West 18th Avenue, Columbus, OH 43210, USA

${ }^{35}$ Physics Department and Tsinghua Center for Astrophysics (THCA), Tsinghua University, Beijing, 100084, People's Republic of China

${ }^{36}$ Las Cumbres Observatory, 6740 Cortona Dr Ste 102, Goleta, CA 93117-5575, USA

${ }^{37}$ Yunnan Astronomical Observatory of China, Chinese Academy of Sciences, Kunming, 650011, People's Republic of China

${ }^{38}$ Key Laboratory for the Structure and Evolution of Celestial Objects, Chinese Academy of Sciences, Kunming 650216, People's Republic of China

${ }^{39}$ Center for Astronomical Mega-Science, Chinese Academy of Sciences, 20A Datun Road, Chaoyang District, Beijing, 100012, People's Republic of China

${ }^{40}$ School of Science, Tianjin University of Technology, Tianjin, 300384, People's Republic of China

${ }^{41}$ Department of Astronomy, University of Texas at Austin, Austin, TX 78712, USA

${ }^{42}$ National Astronomical Observatory of China, Chinese Academy of Sciences, Beijing, 100012, People's Republic of China

${ }^{43}$ Chinese Academy of Sciences South America Center for Astronomy, China-Chile Joint Center for Astronomy, Camino El Observatorio 1515, Las Condes, Santiago, Chile

${ }^{44}$ Homer L. Dodge Department of Physics and Astronomy, University of Oklahoma, Norman, OK, USA

${ }^{45}$ Purple Mountain Observatory, Chinese Academy of Sciences, Nanjing 210034, People's Republic of China

${ }^{46}$ George P. and Cynthia Woods Mitchell Institute for Fundamental Physics \& Astronomy, Texas A. \& M. University, Department of Physics and Astronomy, 4242

TAMU, College Station, TX 77843, USA
${ }^{47}$ Department of Astronomy, School of Physics and Astronomy, Shanghai Jiao Tong University, Shanghai, 200240, People's Republic of China ${ }^{8}$ Department of Physics, University of California, Santa Barbara, CA 93106-9530, USA

49 The Raymond and Beverly Sackler School of Physics and Astronomy, Tel Aviv University, Tel Aviv 69978, Israel

${ }_{50}$ Department of Physics, University of California, 1 Shields Avenue, Davis, CA 95616-5270, USA

${ }^{51}$ LSST, 950 North Cherry Avenue, Tucson, AZ 85719, USA

${ }^{52}$ Konkoly Observatory, MTA CSFK, Konkoly Thege M. ut 15-17, Budapest, 1121, Hungary

${ }^{53}$ Department of Optics \& Quantum Electronics, University of Szeged, Dom ter 9, Szeged, 6720 Hungary

${ }^{54}$ MTA CSFK Lendület Near-Field Cosmology Research Group, Budapest, Hungary

55 Berzsenyi Dániel High School, Kárpát utca 49-53, Budapest, 1133, Hungary

${ }^{56}$ Department of Physics, Lancaster University, Lancaster LA1 4YB, UK

${ }_{58}^{57}$ Institute of Physics, Humboldt-Universität zu Berlin, Newtonstr. 15, D-12489 Berlin, Germany

${ }^{58}$ Institute of Cosmology and Gravitation, University of Portsmouth, Portsmouth PO1 3FX, UK

${ }^{59}$ PITT PACC, Department of Physics and Astronomy, University of Pittsburgh, Pittsburgh, PA 15260, USA

${ }^{60}$ Department of Physics and Astronomy, University of Southampton, Southampton SO17 1BJ, UK

${ }^{61}$ Steward Observatory, University of Arizona, 933 North Cherry Avenue, Room N204, Tucson, AZ 85721-0065, USA

Received 2018 July 30; revised 2018 August 28; accepted 2018 August 31; published 2018 December 28

\begin{abstract}
We present an exquisite 30 minute cadence Kepler (K2) light curve of the Type Ia supernova (SN Ia) 2018oh (ASASSN-18bt), starting weeks before explosion, covering the moment of explosion and the subsequent rise, and continuing past peak brightness. These data are supplemented by multi-color Panoramic Survey Telescope (PanSTARRS1) and Rapid Response System 1 and Cerro Tololo Inter-American Observatory 4 m Dark Energy Camera (CTIO 4-m DECam) observations obtained within hours of explosion. The K2 light curve has an unusual twocomponent shape, where the flux rises with a steep linear gradient for the first few days, followed by a quadratic rise as seen for typical supernovae (SNe) Ia. This "flux excess" relative to canonical SN Ia behavior is confirmed in our $i$-band light curve, and furthermore, SN 2018oh is especially blue during the early epochs. The flux excess peaks $2.14 \pm 0.04$ days after explosion, has a FWHM of $3.12 \pm 0.04$ days, a blackbody temperature of $T=17,500_{-9,000}^{+11,500} \mathrm{~K}$, a peak luminosity of $4.3 \pm 0.2 \times 10^{37} \mathrm{erg} \mathrm{s}^{-1}$, and a total integrated energy of $1.27 \pm 0.01 \times 10^{43} \mathrm{erg}$. We compare SN 2018oh to several models that may provide additional heating at early times, including collision with a companion and a shallow concentration of radioactive nickel. While all of these models generally reproduce the early K2 light curve shape, we slightly favor a companion interaction, at a distance of $\sim 2 \times 10^{12} \mathrm{~cm}$ based on our early color measurements, although the exact distance depends on the uncertain viewing angle. Additional confirmation of a companion interaction in future modeling and observations of SN 2018oh would provide strong support for a single-degenerate progenitor system.
\end{abstract}

Key words: supernovae: general - supernovae: individual (SN 2018oh)

Supporting material: machine-readable table

\footnotetext{
${ }^{62}$ Hubble Fellow, Dunlap Fellow.

${ }^{63}$ Einstein Fellow.
} 


\section{Introduction}

Through a combination of theoretical arguments and strong observational constraints, it has long been understood that Type Ia supernovae ( $\mathrm{SNe} \mathrm{Ia}$ ) are the result of a thermonuclear explosion of a carbon/oxygen white dwarf (WD; e.g., Hoyle \& Fowler 1960; Colgate \& McKee 1969; Woosley et al. 1986; Bloom et al. 2012) in a binary system. Nevertheless, despite SNe Ia being used to discover the accelerating expansion of the Universe two decades ago (Riess et al. 1998; Perlmutter et al. 1999) and continuing to be a powerful dark energy probe (e.g., Jones et al. 2018; Scolnic et al. 2018), we still do not know the nature of their progenitor systems, whether they come from multiple progenitor scenarios, and if so, in what proportion.

Roughly speaking, possible SN progenitor systems can be separated into two main classes (or channels): the singledegenerate (SD) channel, where the primary WD accretes material from a non-degenerate companion triggering a thermonuclear runaway near the Chandrasekhar mass $\left(M_{\mathrm{Ch}}\right.$; e.g., Whelan \& Iben 1973), and the double-degenerate (DD) channel where the SN is triggered by the merger of two WDs (e.g.; Iben \& Tutukov 1984). Numerical modeling of explosions (e.g., Hillebrandt \& Niemeyer 2000; Hillebrandt et al. 2013) combined with radiative hydrodynamic modeling (e.g., Kasen et al. 2009; Woosley \& Kasen 2011; Pakmor et al. 2012; Sim et al. 2012, 2013) indicate that the basic properties of the SN Ia population can be reproduced by either scenario. Therefore, we must turn to observations to further constrain the possible progenitor systems of SNe Ia.

Thus far, the observations have been similarly limited, and are often inconsistent with a single scenario. No SN Ia progenitor system has yet been directly observed in the handful of SNe Ia with reasonably deep pre-explosion images ( $\mathrm{Li}$ et al. 2018; Goobar et al. 2014; Kelly et al. 2014; although one has for a peculiar WD SN, the SN Iax 2012Z; McCully et al. 2014). However, the images were not sufficiently deep to exclude all SD progenitor systems. On a different approach, a search for the surviving non-degenerate companion star at the central regions of SN remnants (SNRs), believed to have a SN Ia origin, also excludes WD + subgiant or red giant (RG) systems (Kerzendorf et al. 2012, 2014; Schaefer \& Pagnotta 2012). Nonetheless, several indirect observations can reveal the nature of the companion with some scenarios having specific and distinct observational predictions.

Observing $\mathrm{SNe}$ Ia as close to explosion as possible can provide unique information for distinguishing between progenitor scenarios. For example, the earliest moments can be dominated by the shock cooling of the exploding WD (Piro et al. 2010), which was used in the case of SN 2011fe to constrain the explosion to be coming from a degenerate star (Bloom et al. 2012). For SD progenitor systems containing a Roche-lobe-filling companion, signs of the SN ejecta interacting with the non-degenerate companion star are expected for some lines of sight (Kasen 2010). This produces strong X-ray and ultraviolet (UV)/optical emission that will surpass the radioactive luminosity of the $\mathrm{SN}$ at these early epochs. The amount of observed flux depends on the viewing angle and the distance between the exploding WD and the companionwhich given the Roche-lobe overflow assumption, provides constraints on the companion star radius. Specifically, evolved RGs are expected to produce more flux than smaller stars.

While early excess emission is a robust prediction for the Roche-lobe-filling SD scenarios, other physical phenomena can possibly also produce early heating. In particular, ${ }^{56} \mathrm{Ni}$ near the surface (i.e., with a mass fraction exceeding that of lower layers) should also introduce flux in excess of the canonical "expanding fireball" model (Piro \& Nakar 2013). This scenario, which can occur for both progenitor channels, can conceal or resemble interaction models. A specific explosion model that can produce such a configuration is the double-detonation explosion of a sub- $M_{\mathrm{ch}} \mathrm{WD}$, where the detonation of a surface helium layer will produce significant surface ${ }^{56} \mathrm{Ni}$ (Noebauer et al. 2017). Piro \& Morozova (2016) also found that shallow ${ }^{56} \mathrm{Ni}$ distributions and/or interaction with circumstellar material (CSM) expelled during a DD merger can modulate the early light curve shape.

Observations that are early enough and with sufficient cadence to search for these early light curve features are still relatively rare. Nearby events, such as SNe 2011fe (Nugent et al. 2011; Bloom et al. 2012), 2014J (Goobar et al. 2014), and ASASSN-14lp (Shappee et al. 2016) provide upper limits to the potential separation distance of the companion, ruling out stars that are more evolved than a RG, while for SN 2009ig, a small blue excess is attributed to the unusual color evolution of the particular event (Foley et al. 2012a). SN Ia sample studies (Hayden et al. 2010a; Bianco et al. 2011; Ganeshalingam et al. 2011; Tucker 2011; Brown et al. 2012) exclude RGs for a fraction of the events, allowing less-evolved stars as companions.

On the other hand, two SNe, SNe 2012cg and 2017cbv, have early light curves that are somewhat consistent with interaction with a companion star. Marion et al. (2016) found that interaction with a $6 M_{\odot}$ main sequence (MS) star can explain the early UV/optical excess of SN 2012cg. For SN 2017cbv, Hosseinzadeh et al. (2017), analyzing many possibilities, favored an interaction with a subgiant companion, within the uncertainties in the modeling. Both of these interpretations have been questioned by Shappee et al. (2018a) and Sand et al. (2018), respectively, where the authors disfavored a nondegenerate companion, based in part on nondetections of stripped hydrogen or helium (within some limits) in nebular spectra. For SN 2012fr, Contreras et al. (2018) found an initial slow, nearly linear rise in luminosity, followed by a faster rising phase, and attributed it to a moderate amount of ${ }^{56} \mathrm{Ni}$ mixing in the ejecta, while for the almost-linear rise of iPTF 16abc, Miller et al. (2018) argued in favor of either ejectamixing or pulsational delayed-detonation models. In the case of the SN 2002es-like (Ganeshalingam et al. 2012) iPTF 14atg (Cao et al. 2016), data are compatible with a companion at a separation of $70 R_{\odot}$; Kromer et al. (2016), using numerical simulations of explosion models, found difficulties reconciling its peculiar spectral evolution with a non-degenerate companion. Finally, Jiang et al. (2017) showed an early red flux excess for MUSSES1604D and, comparing different scenarios, favor a double detonation.

To search for such companion-shock emission, one would ideally conduct a survey with continuous, high-cadence observations to precisely constrain the explosion time and either track or constrain any possible early-time excess flux. The Kepler telescope (Haas et al. 2010), with its wide field of view and 30 minute cadence continuous observations, is particularly well suited to discover $\mathrm{SNe}$ within moments of explosion and continuously monitor those $\mathrm{SNe}$ (for recent transient studies with Kepler see Garnavich et al. 2016; Rest et al. 2018a). Kepler has the ability to observe thousands of galaxies at a time and therefore has the potential to discover 
$\sim 10$ SNe a month if the observations are devoted to relatively nearby galaxies. During the main Kepler mission, Olling et al. (2015) discovered three likely SNe Ia with extraordinary coverage from the moments of explosion through the rise and decline of the SNe. Despite these extraordinary observations, there was no significant detection of interaction.

Nevertheless, it has been demonstrated that Kepler has unique capabilities for precise monitoring of the earliest phases after an SN explosion. To this end, the successor of the Kepler mission, K2, has dedicated a substantial number of targets during Campaign 16, lasting from 2017 December 7 to 2018 February 25, to the K2 Supernova Cosmology Experiment (K2 SCE). Significant advantages of K2 SCE over previous Kepler mission SN studies include (1) monitoring about 50 times as many galaxies (although for a shorter time) and (2) being "forward-facing," where the field is pointed roughly away from the Sun, allowing for simultaneous ground-based observations of all transients discovered in the Campaign 16 field.

In this Letter, we present observations of SN 2018oh, a normal SN Ia whose host galaxy was monitored by the K2 SCE starting before explosion, continuing to first light, and through peak brightness. In addition to its impressive K2 light curve, SN 2018oh SN was extensively monitored by many groundbased facilities. In this Letter, we focus on the first week after explosion. In the data, we robustly identify, with unprecedented photometric coverage, an excess early-time rise component.

This Letter is part of a series of papers analyzing SN 2018oh: Shappee et al. $2018 \mathrm{~b}$ provide an alternative analysis of the $\mathrm{K} 2$ light curve data of the $\mathrm{SN}$ and $\mathrm{Li}$ et al. (2018) presented the photometric and spectroscopic properties of the SN near and after peak brightness.

This Letter is organized as follows. In Section 2, we present the discovery of SN 2018oh and the early-time data that we use in this Letter, including the reduction and calibration steps. In Section 3, we describe the analysis of the early-time light curve, while in Section 4 we propose various physical models that explain it. Finally, in Section 5, we discuss our findings in the context of the progenitor problem of $\mathrm{SNe}$ Ia, and outline our conclusions.

Throughout this Letter, Modified Julian Days (MJDs) are reported as observed days while phases are reported in rest frame, unless where noted. We adopt the $\mathrm{AB}$ magnitude system, unless where noted, and a Hubble constant of $H_{0}=73 \mathrm{~km} \mathrm{~s}^{-1} \mathrm{Mpc}^{-1}$.

\section{Discovery and Early-time Observations}

SN 2018oh was discovered by the All Sky Automated Survey for SuperNovae (ASAS-SN; Shappee et al. 2014) in images obtained on 2018 February 4.41 UT (all times presented are UT; with discovery name ASASSN-18bt; Brown et al. 2018; Shappee et al. 2018b), at $V=15.2 \mathrm{mag}$, with the last nondetections at 2018 January 27.13. The supernova is located at $\alpha=09^{\mathrm{h}} 06^{\mathrm{m}} 39$ s. $592, \delta=+19^{\circ} 20^{\prime} 17^{\prime \prime} .47$ (J2000.0; Cornect et al. 2018), 7!' 8 north and 2!" 0 east of the center of UGC 4780 , an Sdm star-forming galaxy, with a redshift of $z=0.010981$ and a distance of $49.4 \mathrm{Mpc}$. The Milky Way reddening toward SN 2018oh is $E(B-V)=0.0368$ mag (Schlafly \& Finkbeiner 2011). The transient was classified on 2018 February 5 as a relatively young ( -8 to -6 days relative to peak brightness) normal SN Ia (Leadbeater 2018; Zhang et al. 2018).

UGC 4780 was included as a Campaign 16 target through "The K2 ExtraGalactic Survey (KEGS) for Transients" (PI: A. Rest) and the "Multi-Observatory Monitoring of K2
Supernovae" (PI: R. J. Foley) programs as part of the K2 SCE (internal Kepler ID 228682548). After the end of Campaign 16, the data were transferred to MAST, from which we retrieved the UGC 4780 data. We produced a provisional light curve with the "quick look" routine KADENZA 64 (Barentsen \& Cardoso 2018) by summing counts in a $5 \times 5$ pixel aperture centered at the peak of each 30 minute image. The background was determined by estimating the median flux of the outermost pixels. Due to its unique observing strategy, which requires regular thruster use to maintain pointing, K2 data suffer from a "sawtooth pattern" and long-term sensitivity trends, partly due to temperature changes as the Sun angle and the zodiacal light levels change during a Campaign. In order to correct for these effects, third-order polynomials were fit in both spatial dimensions to remove the "sawtooth." To account for the long-term trends, we performed a principal-component analysis that represents the common simultaneous trends seen in the light curves of all the (assumed non-varying) galaxies observed on the same chip. Through an iterative procedure, the optimal number of principal-component analysis vectors was determined to be only one. We then determined and removed the long-term trend for SN2018oh. Finally, the noise was estimated by computing the rms variation just before the explosion and then scaling this by the square root of the galaxy flux plus the SN flux in the aperture. For a more detailed discussion on the K2 reduction steps, see Shaya et al. (2015).

During Campaign 16, we actively observed the K2 field with both the Panoramic Survey Telescope and Rapid Response System 1 (Pan-STARRS1) telescope (PS1; Chambers et al. 2016; Magnier et al. 2016; Waters et al. 2016) and the Cerro Tololo Inter-American Observatory (CTIO) $4 \mathrm{~m}$ Mayall telescope with the Dark Energy Camera (DECam; Honscheid et al. 2008; Flaugher et al. 2015). The main goal was to discover and obtain multi-color light curves of transients in K2-observed galaxies. This program was successful where we discovered nine and eight such transients in C16 with PS1 (Smith et al. 2018) and DECam (Rest 2018; Rest et al. 2018b), respectively. Unfortunately, immediately after the explosion of SN 2018oh, poor weather prevented observations for seven nights. During that gap, we did not have scheduled DECam nights either.

All PS1 and DECam images were reduced using the PHOTPIPE imaging and photometry package (Rest et al. 2005, 2014), which performs standard reduction processes, including bias subtraction, cross-talk corrections, flat-fielding, astrometric calibration, and image resampling. Instrumental point-spread function (PSF) magnitudes are calculated by using DOPHOT (Schechter et al. 1993) on the difference images, and the final calibration is performed with PS1 standard-star fields. This photometric procedure is well tested and has been applied in many transient studies (e.g., Rest et al. 2014; Kilpatrick et al. 2018). We present PS1 and DECam images from before and immediately after explosion, as well as images near peak brightness, in Figure 1.

Spectroscopic and photometric follow-up observations of the SN were performed immediately after its discovery, and a complete presentation of the $\mathrm{SN}$ properties is presented in $\mathrm{Li}$ et al. (2018).

\footnotetext{
${ }^{64}$ https://github.com/KeplerGO/kadenza
} 

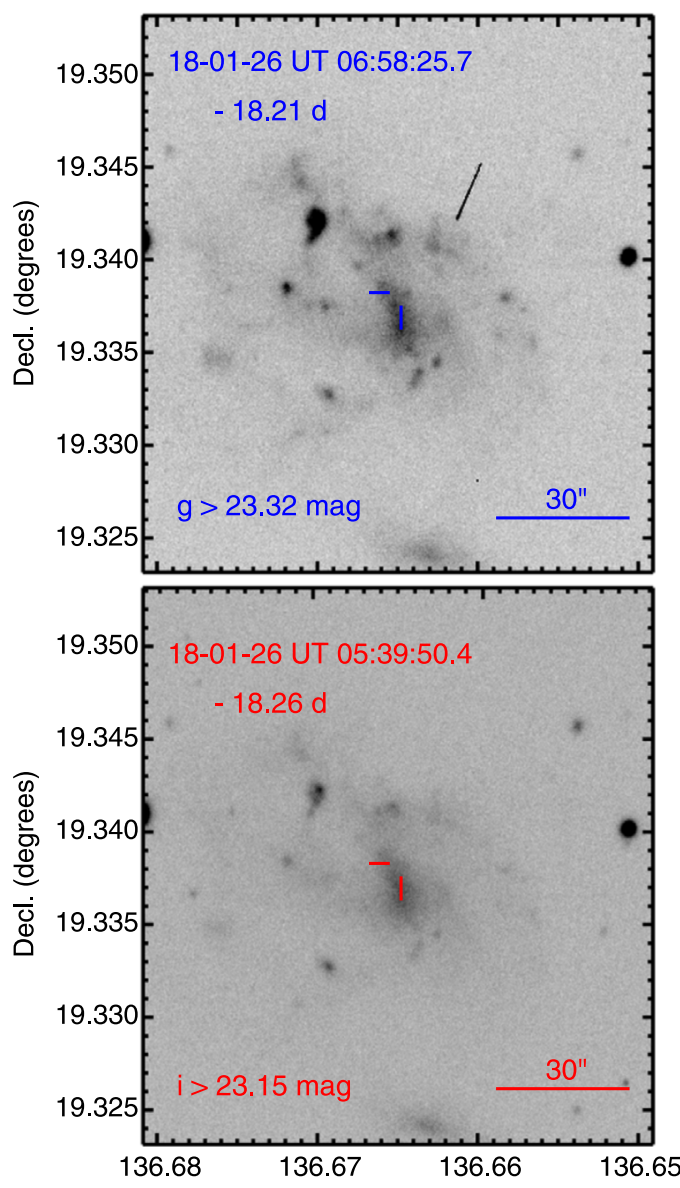
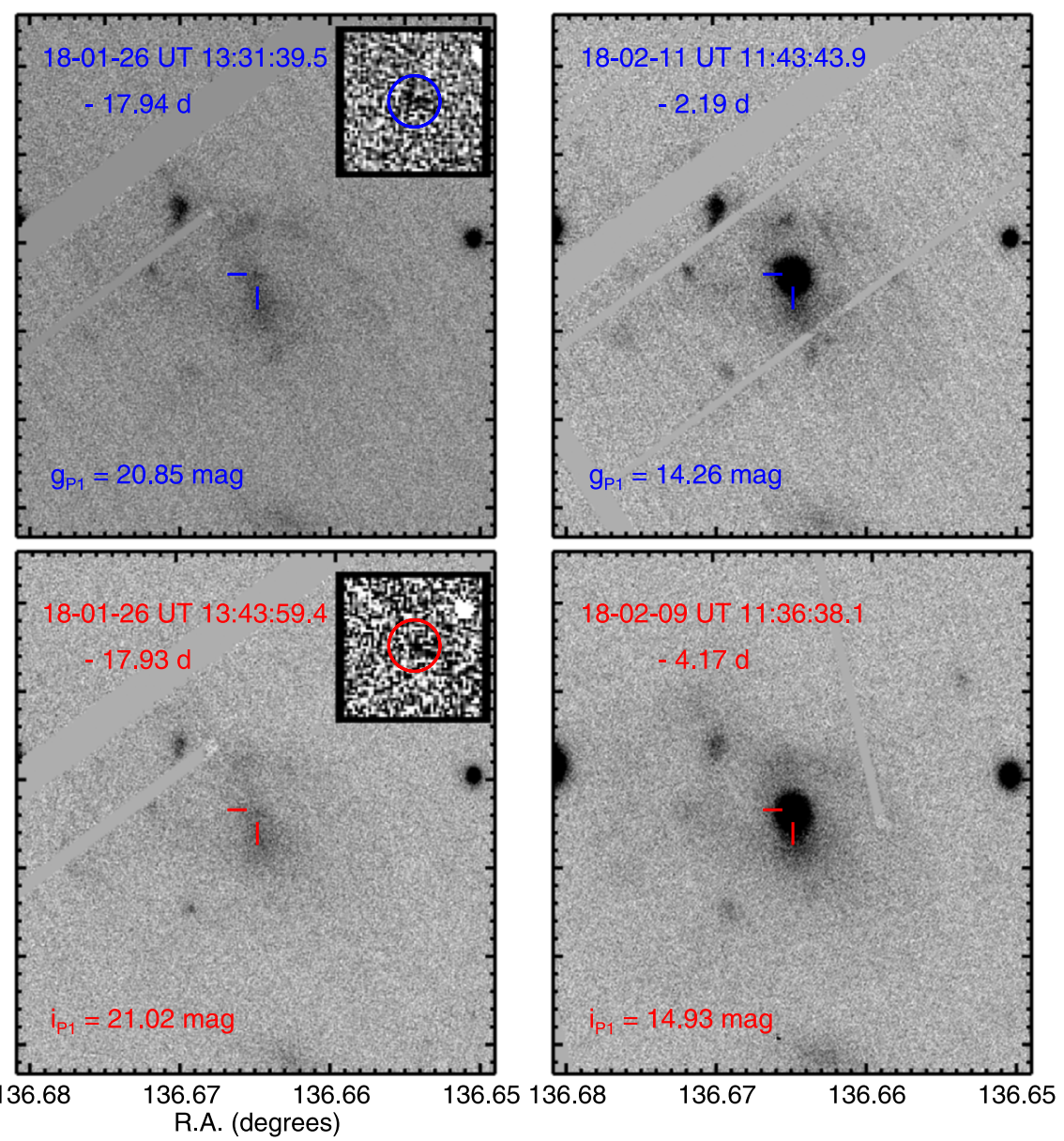

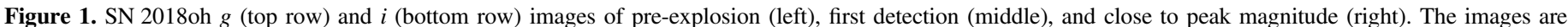

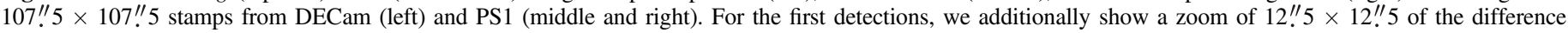

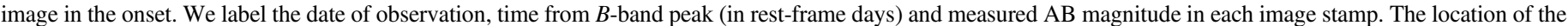
$\mathrm{SN}$ is indicated with a check mark (and a circle for the difference image).

\section{Analysis}

In this section, we present the early photometric observations of SN 2018oh, both from ground-based facilities and K2. We then present a basic analysis of the early evolution of the SN, based on analytical models.

\subsection{Ground-based Photometry}

SN 2018oh is detected in PS1 $g$ and $i$ images, on UT 2018-0126.56 and 26.57 (for $g$ and $i$, respectively), 8.9 days before the ASAS-SN discovery image, with AB magnitudes of $g_{\mathrm{P} 1}=$ $20.72 \pm 0.18$ and $i_{\mathrm{P} 1}=20.94 \pm 0.25$, while the last nondetections were at UT 2018-01-23.38 and UT 2018-01-22.55. Moreover, from DECam $i$-band images taken one day later, SN 2018oh was $i=19.04 \pm 0.01$ and $18.96 \pm 0.01 \mathrm{mag}$ on 2018 January 27.25 and 27.29 , respectively, revealing a rise in the $i$-band of $\sim 1$ mag in one day. A collection of ground-based images, showing pre-explosion, first detection, and close-to-peak luminosity, in $g$ - and $i$-bands, is presented in Figure 1, and reported in Table 1.

After correcting for the Milky Way extinction using the Fitzpatrick (1999) law with $R_{V}=3.1$, we fit the $u B V g r i z$ photometry ( $\mathrm{Li}$ et al. 2018) with the most recent version of the SALT2 light curve fitter (SALT2.4; Guy et al. 2010; Betoule et al. 2014) through the SNANA framework (Kessler et al. 2009). We measure a SALT2 shape parameter of $x_{1}=0.879 \pm 0.012$ and a color parameter of $c=-0.09 \pm$ 0.01 . We determine that $\mathrm{SN} 2018 \mathrm{oh}$ peaked at $B_{\text {peak }}=$ $14.185 \pm 0.010 \mathrm{mag}$ on MJD $58163.339 \pm 0.016$.

To infer the distance, we use the distance estimator from Betoule et al. (2014), and references therein:

$$
\mu=m_{B}-M_{B}+\alpha \times x_{1}-\beta \times c+\Delta_{M},
$$

where $m_{B}, x_{1}$ and $c$ are given above. We use the values of the nuisance parameters $\alpha=0.141, \beta=3.099$, and $M_{B}=-19.17$ given by Betoule et al. (2014). Regarding the host galaxy mass step $\Delta_{M}$ (Kelly et al. 2010; Lampeitl et al. 2010; Sullivan et al. 2010), we use Sloan Digital Sky Survey (SDSS) $g$ and $i$ magnitudes with the relation of Taylor et al. (2011, their Equation (8)) to derive the host galaxy mass of UGC 4780 . We find the mass to be $8.81 \mathrm{dex}$, comfortably on the low-mass side of the step function, and we correct with $\Delta_{M}=-0.06 \mathrm{mag}$. The final distance modulus, assuming $H_{0}=73 \mathrm{~km} \mathrm{~s}^{-1} \mathrm{Mpc}^{-1}$, is estimated to be $\mu=33.61 \pm 0.05 \mathrm{mag}$, corresponding to a distance of $52.7 \pm 1.2 \mathrm{Mpc}$. As UGC 4780 is not in the Hubble flow and has no independent distance measurement, the distance using the $\mathrm{SN}$ itself is the most accurate and precise distance, and we use this distance for the remainder of the analysis.

The near-peak and post-peak photometric data of SN 2018oh show that the SN is a normal SN Ia, while the only spectral 


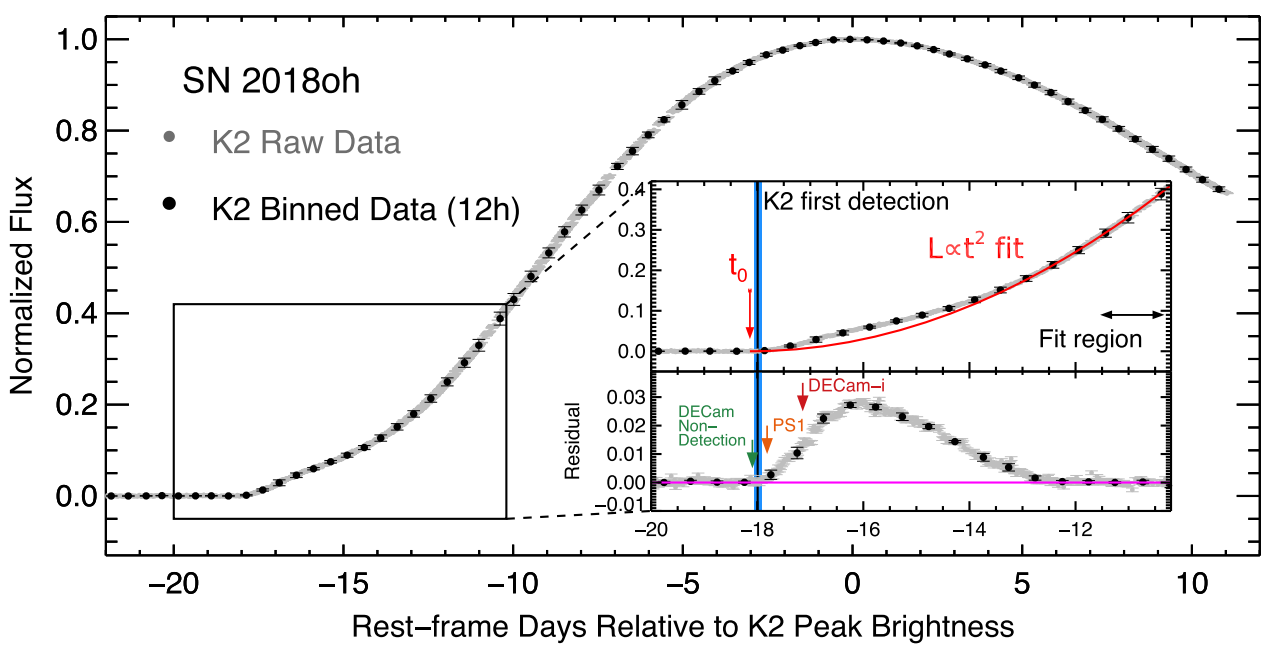

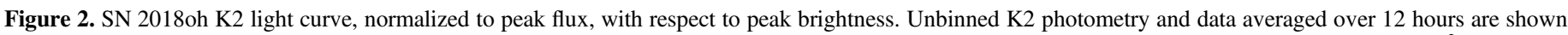

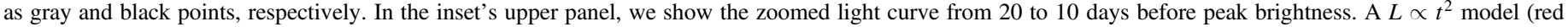

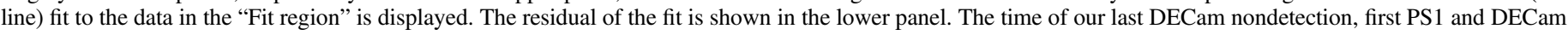

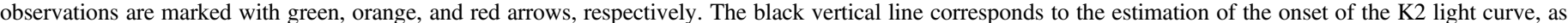
described in the text, with the blue-shaded region representing the $3 \sigma$ standard deviation.

peculiarity is the (relatively) long-lived carbon absorption features, seen even to about three weeks after the maximum light and discussed in $\mathrm{Li}$ et al. (2018). From all available data, we conclude that SN 2018oh is a normal SN Ia.

\subsection{Kepler Light Curve}

After the reduction of the SN 2018oh Kepler/K2 light curve as described in Section 2, which only provides a relative-flux light curve, we determine the true $\mathrm{K} 2$ flux as follows. We use the uBVgriz photometry ( $\mathrm{Li}$ et al. 2018), which has been calibrated to the PS1 system to determine the SN 2018oh flux as a function of time and wavelength. We then use the "max model" of the SNoOPy ${ }^{65}$ package (Burns et al. 2015) to determine the spectral energy distribution (SED) of the SN as a function of time. This model first fits for the peak flux in each photometric band by scaling template light curves (Burns et al. 2011) to the data, with the model $K$-corrections calculated by warping the Hsiao et al. (2007) SN Ia spectral series to match the observed colors. This approach accounts for assumptions about host-reddening and the distance to the $\mathrm{SN}$ by modeling the multi-band photometry before determining the $\mathrm{K} 2$ magnitudes. The best-fit parameters were used to normalize the mangled spectral series to the observed photometry and to generate a synthetic SED. As the Burns et al. (2011) method mangles the spectral series to match the SED in each observerframe passband, there is a choice of which passband's normalized SED could be used as a model for the K2 band. We use the $V$-band as its effective wavelength is closest to that of the K2 band. After integrating over the K2 passband, recovering the "synthetic" K2 light curve, we solve for the absolute zeropoint, using the background-subtracted K2 flux light curve, interpolated over a range of $\pm 3 \mathrm{~d}$ around the time of $B$-band maximum light, where the supernova color evolves slowly. We estimate $\mathrm{ZP}_{\mathrm{K} 2}=25.324 \pm 0.004$ (statistical). We find $a \pm 0.011$ (systematic) mag, systematic uncertainty arising from the choice of which (observer-frame) passband normalized SED is used to model the synthetic K2 light curve.

\footnotetext{
65 https://users.obs.carnegiescience.edu/cburns/SNooPyDocs/html/
}

We present the SN 2018oh K2 light curve in Figure 2, normalized to the peak of the light curve, which we estimate by fitting a polynomial to the data from MJD 58160.0 to 58165.0. We find that the peak in the $\mathrm{K} 2$ band occurs at $\mathrm{MJD}_{\max }^{\mathrm{K} 2}=58162.58, \sim 0.12$ days prior to $B$-band maximum, with $\mathrm{K} 2{ }_{\max }=14.401 \pm 0.001$. A portion of the light curve is presented in Table 2, while the complete data set is available in the electronic edition.

\subsection{Basic Analysis of the K2 Light Curve}

Assuming that the photospheric temperature of a SN Ia does not change significantly in the first few days after explosion, the luminosity of the Rayleigh-Jeans tail of the blackbody radiation will increase with time as $\mathrm{L} \propto t^{2}$ (Arnett 1982; Riess et al. 1999), as the size of the photosphere increases. However, the K2 light curve of SN 2018oh shows a prominent "twocomponent rise": an initial flux excess, from $\sim 18$ to 13 days before peak brightness, which eventually subsides and the usual "expanding fireball" rise dominates starting about 13 days before peak brightness.

We determine the onset of the K2 light curve as follows. For a given sliding time window, we calculate the weighted-mean of the flux and we compare it with the flux of the time window prior to it, marking as a detection when $\operatorname{Flux}_{i}>3 \sigma_{i-1}$. By an iterative procedure, using decreasing time-window widths, we record the detection times and we estimate their mean and standard deviation. We calculate $t_{\mathrm{det}}^{\mathrm{K} 2}=-17.99 \pm 0.04$ days from maximum light (at $\mathrm{MJD}_{\mathrm{det}}^{\mathrm{K} 2}=58144.39$ ), shown as the black vertical line in Figure 2. We note that the first PS1 detections were 0.18 days (4.32 hours) after the $\mathrm{K} 2$ first detection, which we estimate to be 2018 January 26.04 .

In order to determine the properties of the power-law rise (i.e., excluding the first component rise), we attempt to estimate a time range by iteratively fitting, using IDL's MPFIT function, a $\left(t-t_{0}\right)^{2}$ power law to the data in a window from a variable (shifting by steps of 0.02 days) start time beginning 20 days before peak brightness until the flux reaches $40 \%$ of the peak flux, as has been done with other Kepler SN Ia studies (Olling et al. 2015). Our best fit (reduced $\chi^{2}=1.09$ ) is for a time 


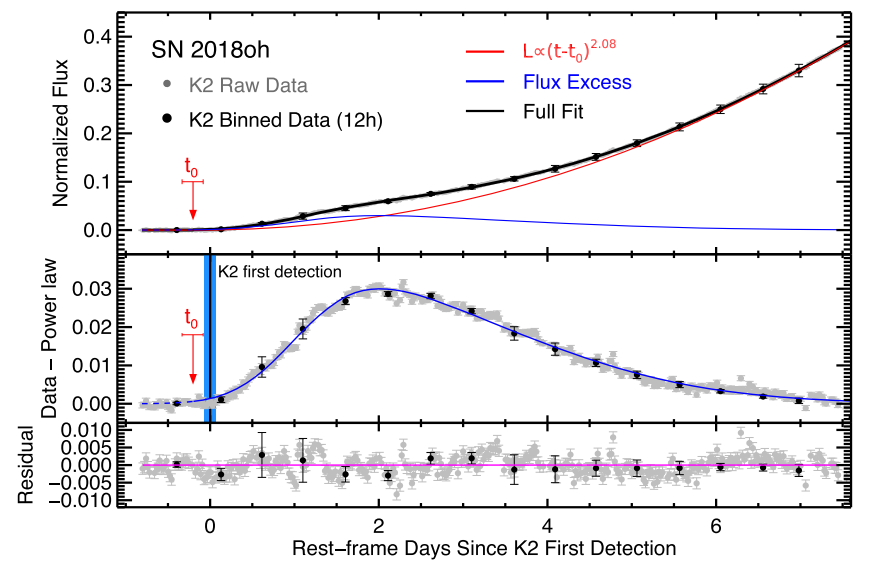

Figure 3. Top: SN 2018oh K2 light curve, normalized to peak flux, with respect to the first $\mathrm{K} 2$ detection. Our full fit is shown as a solid black line, while the decomposition of the fit is shown as a red line for the SN power-law flux, and a blue line for the first rise component. The red downward arrow denotes the time of first light $t_{0}$, estimated from the fit. Middle: the early flux excess, plotted as the data minus the fitted power-law model. The result of the first component fit is shown as a blue line. Bottom: the residual of the fit (data minus full model fit).

window from 11.54 to 10.32 days before peak brightness, which we mark with a vertical two-headed arrow in Figure 2. From this fit, we estimate a time of first light, $t_{0}=-18.14 \pm$ 0.02 days $-\sim 0.15$ days $(\sim 3.6$ hours) before our first K2 detection. We display the residual to the fit in the bottom panel of the inset in Figure 2 . We find that $\sim 2$ days after $t_{0}$, the flux excess is $\sim 3$ times as luminous as the power-law rise, and represents $\sim 65 \%$ of the total flux at that time.

As it has been shown in previous rise time studies (Riess et al. 1999; Hayden et al. 2010b; Ganeshalingam et al. 2011; González-Gaitán et al. 2012; Firth et al. 2015), the index of the power law can significantly vary from 2 for a particular SN. To account for this possibility, we repeat the previous procedure and, using emcee, a Python-based application of an affine invariant Markov chain Monte Carlo (MCMC) with an ensemble sampler (Foreman-Mackey et al. 2013), we fit a $\left(t-t_{0}\right)^{\alpha}$ power law (thus, additionally fitting for the power-law index). Doing so, we find a similar best-fit region as before, with the new best-fit parameters $t_{0}=-17.86_{-0.25}^{+0.24}$ days before peak brightness, with $\alpha=1.92 \pm 0.07$.

In order to quantify the rise of the excess flux component, and motivated by its shape, we consider a simple analytical model that consists of (1) a power law $L \propto\left(t-t_{0}\right)^{\alpha}$ and (2) a skewed Gaussian to account for the early flux excess. We fit the light curve from 20 days before peak brightness through the time when the flux reaches $40 \%$ of the peak flux, with both a fixed power-law index of 2, and with the index allowed to float. By fixing the index to $\alpha=2$, we estimate $t_{0}=-18.00_{-0.02}^{+0.03}$. When simultaneously fitting for the power-law index, we find $t_{0}=-18.19 \pm 0.05$ and $a=2.08 \pm 0.02$. The later fit is shown in Figure 3. These results are generally consistent with the canonical expanding fireball model, and the initial assumption that $L \propto\left(t-t_{0}\right)^{2}$ seems reasonable given the data.

From the multi-component fit, we also estimate that the early excess flux peaked with a luminosity of $(4.3 \pm 0.2) \times$ $10^{37} \mathrm{erg} \mathrm{s}^{-1}$ at $t_{\text {peak }}^{c 1}=-16.05 \pm 0.04$ days, approximately 2.2 days after $t_{0}$, and had a FWHM of 3.12 days. The total emitted energy above the power-law rise is $(1.27 \pm 0.01) \times$ $10^{43} \mathrm{erg}$.
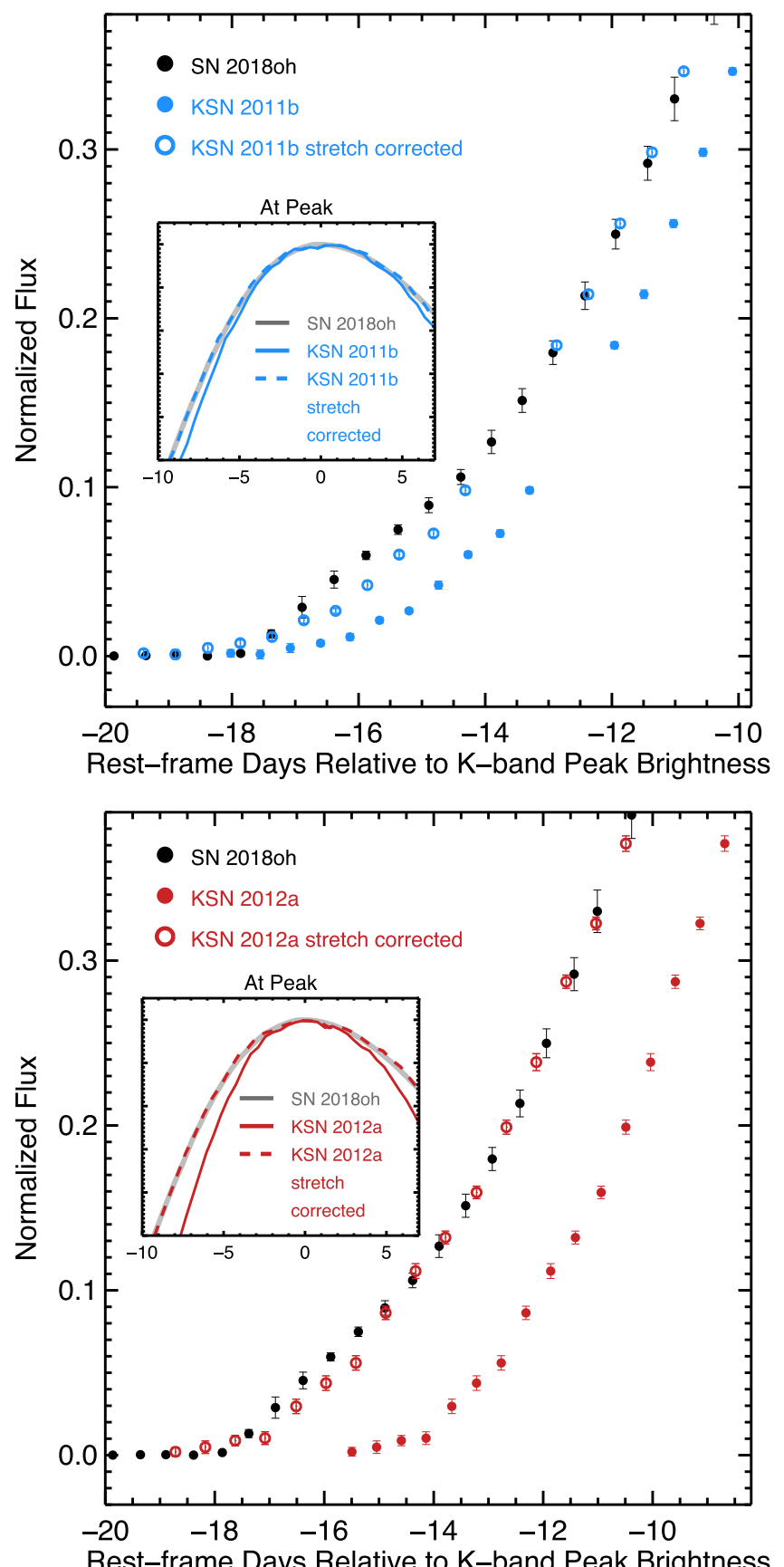

Figure 4. Comparison of the K2 SN 2018oh light curve (black circles), normalized to peak, with respect to peak brightness, with KSN 2011b (upper panel) and KSN 2012a (lower panel). The Kepler light curves of KSN 2011b and KSN 2012a have been "stretch-corrected" to match the SN 2018oh light curve. We show the original $12 \mathrm{hr}$ time binned data from Olling et al. (2015) in full circles, and the "stretch-corrected" ones with open circles. In the insets, we show a zoom of the light curves at peak.

\subsection{Comparison to Other SNe}

First, we compare the K2 light curve of SN 2018oh with the Kepler SNe presented in Olling et al. (2015), focusing on the discovery and rise epochs (Figure 4). As mentioned in Olling et al. (2015), KSN 2011b (blue full circles) and KSN 2012a (red full circles) occurred in red and passive galaxies at redshifts $\sim 0.05$ and $\sim 0.09$ (we exclude the third Kepler $\mathrm{SN}$ of Olling et al. 2015, KSN 2011c, due to the lower quality of data). Moreover, these $\mathrm{SNe}$ are fast decliners (thus, have lower 


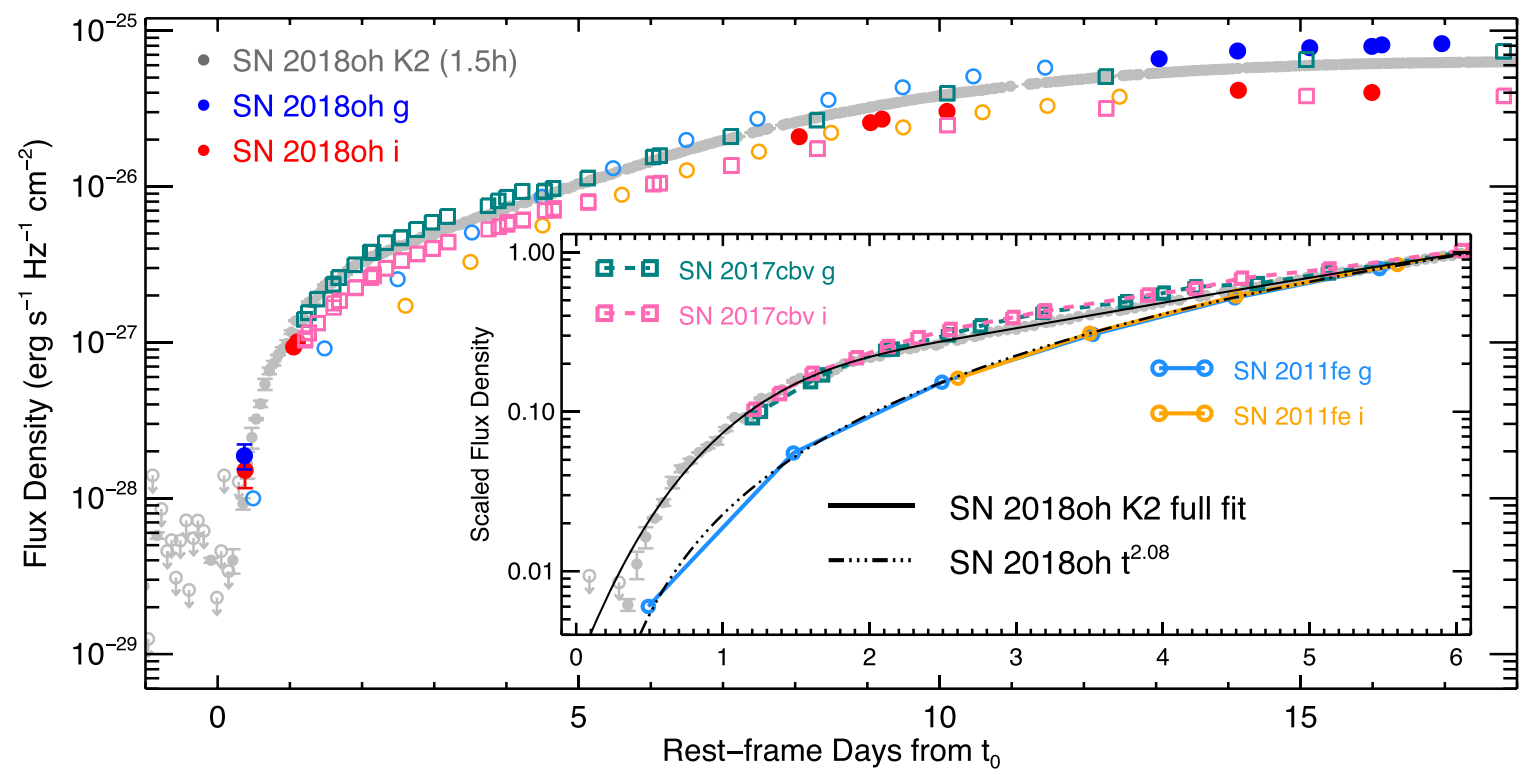

Figure 5. SN 2018oh flux in $g_{\mathrm{P} 1}$ (blue), $i$ and $i_{\mathrm{P} 1}$ (red), and $\mathrm{K} 2$ (gray), with respect to the rest-frame time since first light, $t_{0}$, as estimated in Section 3.3. Nondetections in the K2 band are plotted as open gray circles. Similar light curves for SN 2011fe ( $g$ and synthetic $\left.i_{\mathrm{P} 1}\right)$ and SN 2017cbv $(g$ and $i)$ are also shown, after being normalized to the appropriate peak flux of SN 2018oh. Inset: a zoomed-in region of the extremely early light curve, normalized to the K2 flux at six days after explosion. Note that the comparison SN sample is additionally plotted as solid (SN 2011fe) and dashed (SN 2017cbv) lines, with some of the photometric points removed for clarity. We overplot the fits described in Section 3 as indicated in the legend.

absolute luminosities), while SN 2018oh is a normal SN Ia. For this reason, we "stretch-correct" (Perlmutter et al. 1997) the Kepler SN light curves to the K2 light curve of SN 2018oh by determining the stretch factor that, when applied, best matches the light curves (see the insets in Figure 4). The "stretchcorrected" light curves are shown as open blue (KSN 2011b) and red (KSN 2012a) circles.

As it can be seen, the applied stretch correction successfully matches the $\mathrm{SNe}$ at the epochs around peak brightness. However, SN 2018oh clearly deviates for the first few days after explosion, when the flux excess is observed. We estimate that, at the time of the peak of the flux excess, $t_{\text {peak }}^{c 1}=-16.05$ days, SN 2018oh is $51 \%$ and $32 \%$ more luminous than the stretch-corrected KSN 2011b and KSN 2012a, respectively.

Next, we compare the early SN 2018oh light curves with those of two other SNe with very early data: the well-studied, extremely young SN 2011fe (Nugent et al. 2011), a normal Type Ia supernova that shows no flux excess at the extremely early times, and SN 2017cbv (Hosseinzadeh et al. 2017), an SN Ia with a prominent blue early flux excess. For this comparison, we need comparable filters. SN 2017cbv has extensive early-time photometry in the desired $g$ - and $i$-bands (Hosseinzadeh et al. 2017). SN 2011fe also has an early $g$-band light curve (Nugent et al. 2011), but lacks an early $i$-band light curve. In place of filtered photometry, we use the Pereira et al. (2013) spectrophotometric time series, from which we synthesize an $i$-band light curve.

In Figure 5, we simultaneously display the early SN 2018oh $\mathrm{K} 2$ (gray), $g$ (blue), and $i$ (red) light curves. For comparison, we also show similar data for SNe $2011 \mathrm{fe}$ and $2017 \mathrm{cbv}$. In the inset, we show the first six days after explosion, where the SN rose $>2$ orders of magnitude in flux. We also display the full two-component fit to the SN 2018oh light curve and just the power-law component.

While SN 2011fe clearly lacks the flux excess of SN 2018oh and rises close to $t^{2}$, SN $2017 \mathrm{cbv}$ has a flux excess at early times and an early photometric behavior comparable to
SN 2018oh. At later times $(t>-10$ days), all three $\mathrm{SNe}$ evolve similarly. Notably, from that point on, SN 2018oh looks identical to the "normal" SN 2011fe.

Finally, we investigate the color evolution of SN 2018oh, and in particular the $g-i, g-\mathrm{K} 2$ and $\mathrm{K} 2-i$ colors. We compare the SN2018oh colors to the synthetic colors of SNe 2011fe and 2017cbv, calculated as described above (note that we also estimate the synthetic K2 magnitude). Additionally, we compute the color evolution of the Hsiao et al. (2007) template spectra. The results are shown in Figure 6.

While SNe 2018oh and 2017cbv generally have similar colors for the epochs examined here, generally having bluer colors than both SN 2011fe and the Hsiao et al. (2007) template, there is a distinct difference at the earliest epochs, when the prominent flux excess is observed. We note the difference in the $\mathrm{K} 2-i$ color, at the onset of the excess flux component where SN 2018oh is bluer than all comparison SNe ( $\sim 0.1$ and $0.08 \mathrm{mag}$ from SN 2011fe and SN 2017cbv, respectively). Unfortunately, we only have a single $i$ observation during this phase. Nonetheless, this single observation is critical in separating SN 2018oh from SN 2017cbv.

\subsection{SED of the Excess Flux}

Finally, we investigate the SED of the excess flux observed from 18 to 13 days before peak brightness. While we have no spectra during this phase, we have filtered photometry that can constrain the SED. In addition to the K2 photometry, we will use the PS1 $g$ and DECam $i$ observations at $t=-17.8$ and -17.1 days, respectively, which were obtained while the flux excess was still rising (see the inset of Figure 2).

We will focus on the crucial DECam $i$ observation at $t=-17.1$ days from the $\mathrm{K} 2$ maximum, which coincides with the rise of the flux excess (see inset of Figure 2). While there is no spectrum of SN 2018oh taken at that epoch, motivated by the similar peak/post-peak photometric and spectroscopic behavior with SN2011fe, we use the Lick/KAST spectrum, 

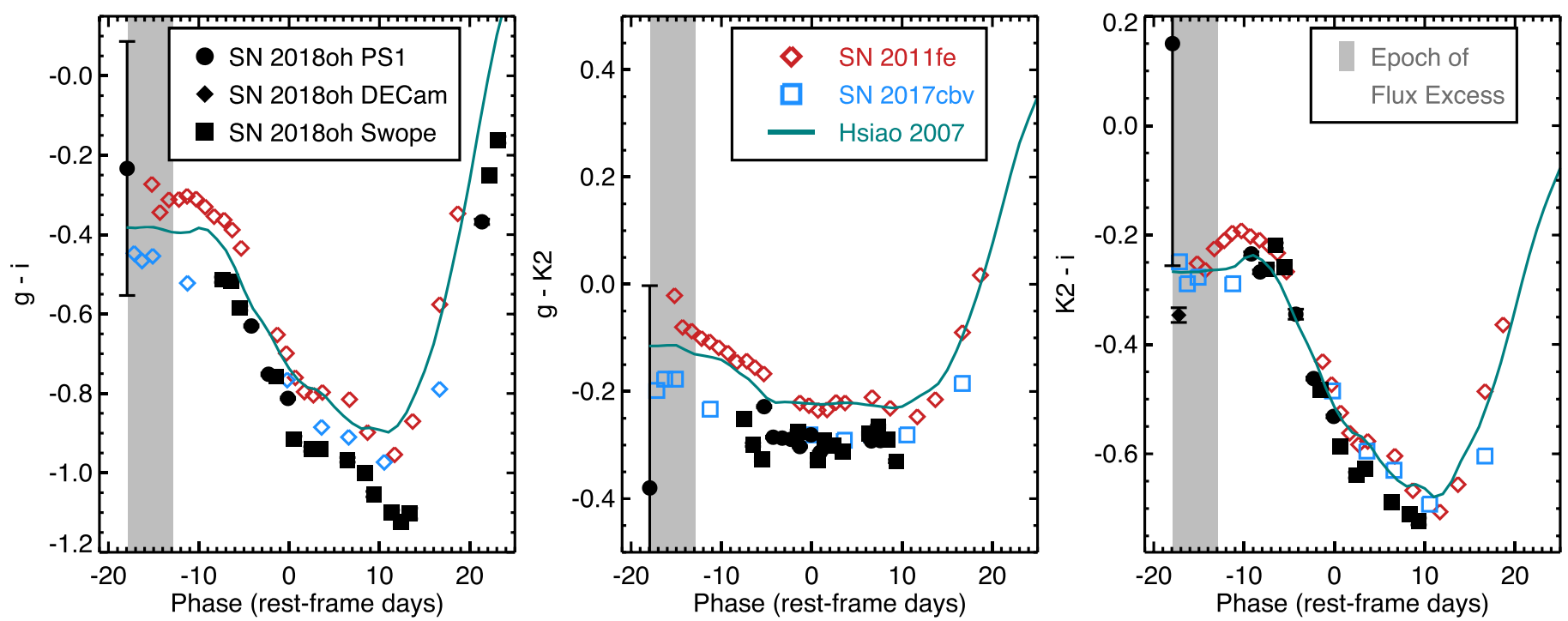

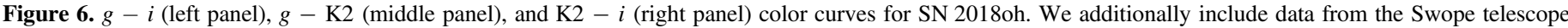

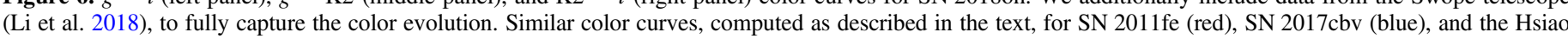
et al. (2007) templates (green) are overplotted. The gray-shaded region corresponds to the duration of the flux excess.

presented initially by Nugent et al. (2011), taken $\sim 1.5$ days after the SN 2011 fe explosion ( -16.33 rest-frame days from $B$-band maximum light). We attempt to spectroscopically match this spectrum (for which no flux excess is observed) with the photometric colors of SN 2018oh at the epoch in question. As mentioned above, we unfortunately do not have $g$ observations at this epoch; therefore, we assume no color evolution in $g-i$ for the first days (see the left panel of Figure 6). We note that this assumption is somehow arbitrary. Our photometry at -17.8 days has large uncertainties, while the $g-i$ color is redder compared to SN 2017cbv. Nevertheless, after redshifting the spectrum to the redshift of SN 2018oh, we scale it to match the SN component of the K2 flux at -17.1 days, as determined in Section 3.3, Figure 3. We then perform a MCMC fit of this spectrum and a blackbody spectrum, where the resulting spectrum reproduces the observed photometry, with the results shown in Figure 7.

Our best fit includes a blackbody with $T=17,500_{-9,000}^{+11,500} \mathrm{~K}$. The main source of the large uncertainty comes from the constant color evolution assumption and the corresponding large photometric uncertainty at this extremely early epoch. However, the resulting fitted temperature is high, providing an indication of a hot blackbody component, on top of the normal SN spectrum.

\section{Models}

We next consider three scenarios that may provide additional heating at early times to lead to the two-component rise seen in the SN 2018oh light curve: the interaction between the SN and a nearby companion star, a double-detonation model with ${ }^{56} \mathrm{Ni}$ near the surface of the star, and an additional model in which we tune that amount of surface ${ }^{56} \mathrm{Ni}$ in an attempt to best match SN 2018oh.

\subsection{Interaction with a Companion Star}

One potential explanation for the early-time excess is shock interaction between the supernova ejecta and a non-degenerate binary companion (Kasen 2010). The collision is characterized by prompt X-ray emission, followed by an optical/UV excess lasting about one week after explosion. Although the excess peaks in the UV, a measurable signature is observable in the Kepler bandpass if the system is configured in a favorable viewing angle (Olling et al. 2015).

To test this scenario, we use a numerical model for the early light curve following the methods outlined in Piro \& Morozova (2016; using the Chandrasekhar progenitor models from Martínez-Rodríguez et al. 2016) that roughly matches the rise of SN 2018oh, and then combine this with the analytic interaction model of Kasen (2010). The interaction emission is mainly controlled through two parameters, the orbital separation $a$ and the characteristic ejecta velocity $v$. Because $a$ can vary by many orders of magnitude and $v$ is relatively well constrained to be $v \approx 10^{9} \mathrm{~cm} \mathrm{~s}^{-1}$, this makes the interaction a powerful diagnostic for measuring $a$. In addition, there are viewing-angle effects, but this is somewhat degenerate with the other parameters. Thus we focus on the the case when one is observing directly into the shocked region (when the companion is roughly between the explosion and the observer) and take the measured $a$ as a lower limit to the orbital separation.

From this procedure, we find that a collision with a companion at $a=2 \times 10^{12} \mathrm{~cm}$ provides a reasonable match to the early rise. We plot this as a solid blue line in the right panel of Figure 8, and also show the constituent parts of the interaction (dashed blue line) and the SN itself (dotted blue line). An important assumption of this model is that the companion is overfilling its Roche lobe, and therefore we can approximate its radius as (Eggleton 1983)

$$
R=\frac{0.49 q^{2 / 3}}{0.6 q^{2 / 3}+\ln \left(1+q^{1 / 3}\right)} a,
$$

where $q$ is the ratio of the companion and WD's mass. For a range of companion masses from $M \approx 1-6 M_{\odot}$, this results in $R \approx 10-15 R_{\odot}$, respectively. This is generally too large for a main sequence star, and thus we conclude that the companion must be a subgiant if interaction is the correct explanation for the early excess. 


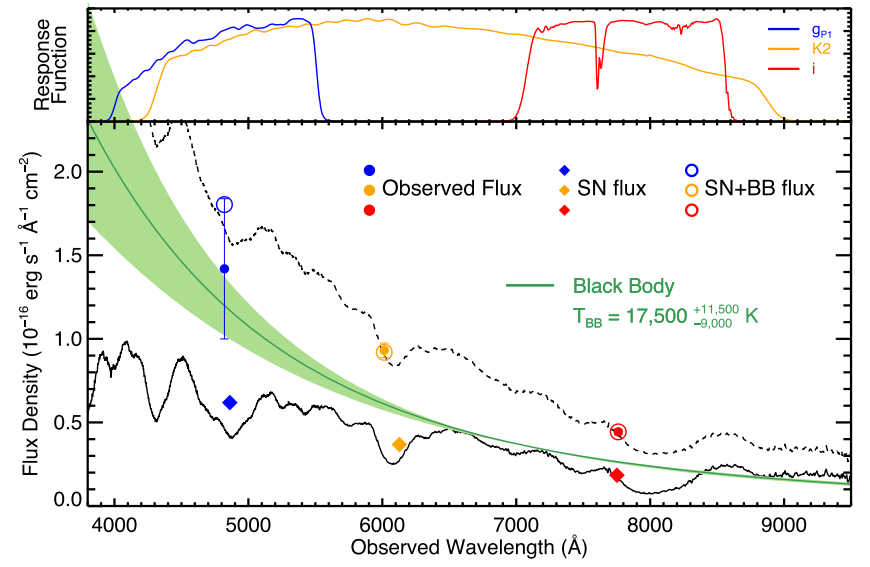

Figure 7. + 1.5 day from explosion SN 2011 fe spectrum (solid black line), redshifted and scaled to match the $t^{2.08} \mathrm{SN}$ component of the SN 2018oh K2 photometry at +1.09 days after $t_{0}$, obtained from the fit shown in Figure 3 . The $g_{\mathrm{P} 1}$ (blue), K2 (orange), and $i$ (red) photometry are shown as full diamonds, at each filter's effective wavelength. The solid green line is the best-fit blackbody spectrum that reproduces the observed photometric colors at +1.09 days after $t_{0}$ (full circles), with the open circles representing the synthetic fluxes of the resulting spectrum (dashed line). In the upper panel, we show the response functions of the $g_{\mathrm{P} 1}$ (blue), K2 (orange) and DECam $i$ (red) filters.

\subsection{Double-detonation Sub-Chandrasekhar Explosion}

Another possible mechanism for creating an early-time flux excess is the double-detonation scenario for exploding a subChandrasekhar mass C/O WD with an accreted shell of helium on its surface. In this scenario, the helium shell detonates, producing on the surface some abundance of radioactive elements such as ${ }^{56} \mathrm{Ni}$ and ${ }^{48} \mathrm{Cr}$, and sending a shockwave into the WD that then ignites the $\mathrm{C} / \mathrm{O}$ core (Woosley \& Weaver 1994). The result produces observables generally consistent with a SN Ia; however, the amount of Fe-group elements synthesized during the He-shell detonation must be small to resemble SNe Ia near peak brightness. The photons produced by the radioactive decay of material on the surface quickly diffuse out of the ejecta, creating a flux excess relative to a typical SN Ia in the first few days after explosion (Noebauer et al. 2017).

We test this scenario as a candidate for SN 2018oh by exploring a hydrodynamic and radiative transfer numerical survey of double-detonations of sub-Chandrasekhar-mass white dwarfs, the results of which will be presented in full by A. Polin et al. (2018, in preparation). The parameter space of the survey spans from 0.7-1.2 $M_{\odot}$ WDs with helium shells from 0.01 to $0.08 M_{\odot}$ and a range of mixing mass from 0.05 to $0.3 M_{\odot}$. The best-fitting model, based on a reduced $\chi^{2}$ measurement, is a $0.98 M_{\odot}$ WD with $0.05 M_{\odot}$ of Helium on its surface, with the ejecta smoothed over a mixing length of $0.25 M_{\odot}$. This model produces a total of $0.448 M_{\odot}$ of ${ }^{56} \mathrm{Ni}$, $3.65 \times 10^{-3} M_{\odot}$ of ${ }^{48} \mathrm{Cr}$, and $1.8 \times 10^{-2} M_{\odot}$ of ${ }^{52} \mathrm{Fe}$. From these elements, the amount of each that is synthesized in the helium shell (i. e., in the outer layers of ejecta) is $1.22 \times 10^{-2}$, $3.19 \times 10^{-2}$, and $6.11 \times 10^{-2} M_{\odot}$, corresponding to $2.7,87.4$, and $33.9 \%$, respectively. The $\mathrm{K} 2$ synthetic light curve is shown in the left panel of Figure 8. The approximate magnitudes of both the early-time excess and peak are reproduced, as is the duration of the excess and rise time to peak brightness.

\subsection{A General Off-center Nickel Distribution}

The previous model is specifically applicable to the DD scenario, but it is possible in principle that other scenarios may $\operatorname{mix}{ }^{56} \mathrm{Ni}$ to the outermost layers. To explore this possibility more generally, we consider models in which we take a normal $\mathrm{SN}$ Ia explosion and place by hand some amount of ${ }^{56} \mathrm{Ni}$ near the surface. As with the supernova model for the interaction scenario, we use the methods outlined in Piro \& Morozova (2016) with the progenitors generated in the work of MartínezRodríguez et al. (2016). Using this we place the ${ }^{56} \mathrm{Ni}$ in two distinct regions, a centrally concentrated region that provides the main rise and a shallow region above a mass coordinate of $1.3 M_{\odot}$. The shallow abundance is varied to find the best fit with the $\mathrm{K} 2$ photometry, including smoothing with a $0.05 M_{\odot}$ boxcar, which prevents numerical issues from sharp compositional gradients.

Our best-fit model under this scenario has $0.03 M_{\odot}$ of ${ }^{56} \mathrm{Ni}$ near the surface of the WD as shown by the orange solid line in the middle panel of Figure 8. The model reproduces the general evolution of the light curve, encapsulating the initial flux excess. We therefore provisionally consider this a viable model. Whether or not such a model can reproduce the full photometric and spectroscopic evolution of SN 2018oh is less clear. Iron-peak elements at shallow depths can provide extensive line-blanketing that alters the colors and spectra of the SN at peak luminosity, potentially making it difficult for SN 2018oh to be a spectroscopically normal SN Ia. Below we consider in further detail whether such a model can even reproduce the early color evolution of SN 2018oh.

\subsection{Detailed Model Comparisons}

Having found both SD and DD models that can reproduce the K2 light curve, we must examine additional data that differentiate these scenarios. The earliest detections by PS1 and DECam are particularly powerful for this purpose.

In addition to detecting the flux excess in the $\mathrm{K} 2$ band, we also detect an excess in the $i$-band (see Figure 5). Examining the $\mathrm{K} 2-i$ color during the flux excess, we find that SN 2018oh is bluer than SN 2011fe by almost 0.2 mag. Moreover, SN 2018oh is also similarly bluer than SN 2017cbv at that epoch. This means that one day after explosion, SN 2018oh is not only distinct from the normal SN $2011 \mathrm{fe}$ but also from SN 2017cbv, which also had an early-time flux excess (Hosseinzadeh et al. 2017). After the onset of the canonical SN rise (rightward of the gray-shaded region in the panels of Figure 6), the three SNe evolve in a similar manner (apart from the usual color dispersion seen in Type Ia supernovae).

All of the models examined above are able to reproduce the flux excess at early times of SN 2018oh, but with two main different physical origins. These models predict very different SEDs and in particular different colors. Specifically the companion-interaction model is expected to be bluer than the surface-Ni model.

We display the expected $g-i, g-\mathrm{K} 2$, and $\mathrm{K} 2-i$ colors for each model in Figure 9. The double-detonation model is particularly red, and it is unable to explain the blue colors of SN 2018oh. Moreover, sub-Chandrasekhar double-detonations also leave spectral signatures such as a significant Ti II absorption features in the peak spectra, which are not seen in 


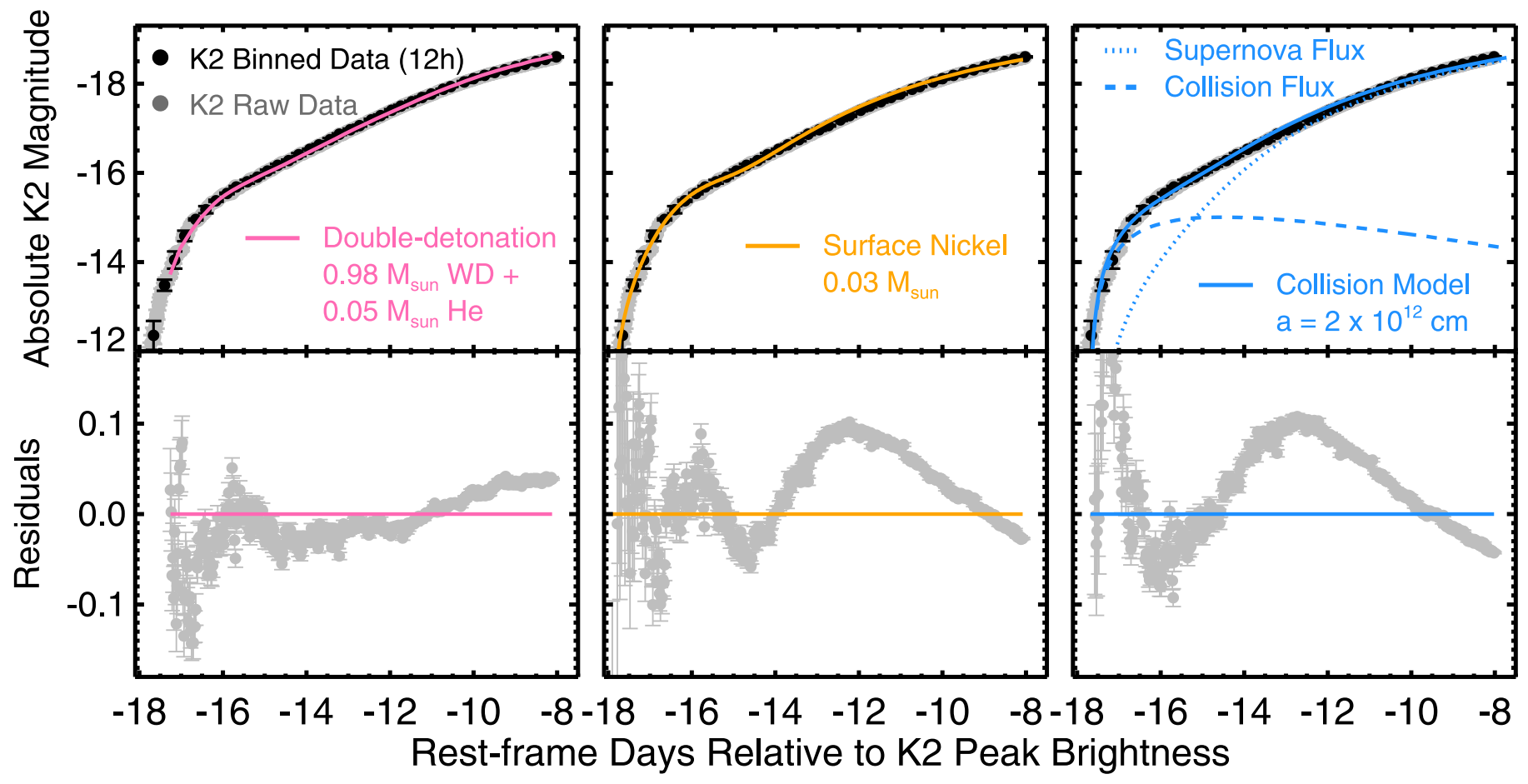

Figure 8. Absolute magnitude K2 early-time light curve of SN 2018oh (gray and black circles). We overplot our best-fit models, as described in Section 4, of the double-detonation of a $0.98 M_{\odot}$ WD with a $0.05 M_{\odot}$ He layer on its surface (left panel, solid pink), of a near-Chandrasekhar mass WD explosion with a surface layer of $0.03 M_{\odot}$ nickel (middle panel, solid orange) and of a collision model (Kasen 2010) with a non-degenerate companion at a distance of $a=2 \times 10^{12} \mathrm{~cm}$ (right panel, solid blue). We show the decomposition of the collision model to the contribution of the SN flux (dotted blue) and the interaction of the SN ejecta with the companion (dashed blue). The residuals of the model fits are shown in the lower panels.
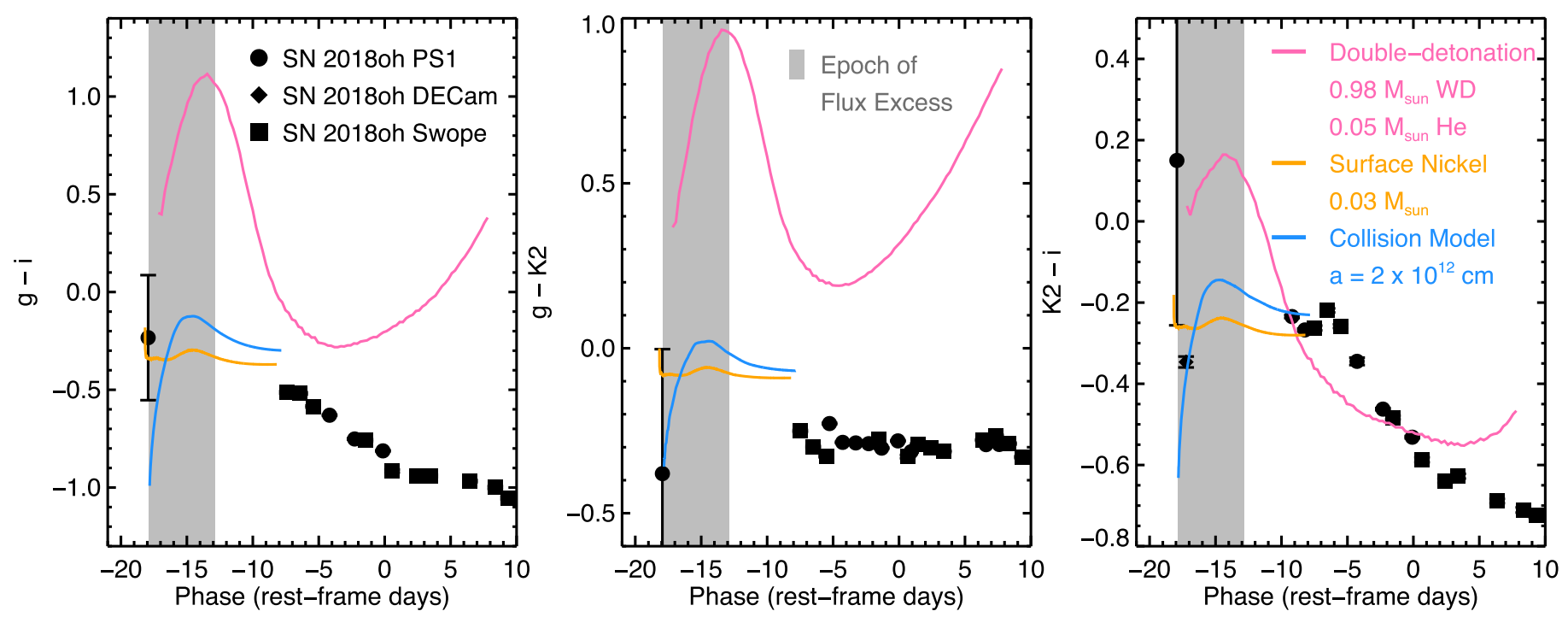

Figure 9. Same as in Figure 6 (note the different ranges in the axes), but comparing with the predicted colors of the double-detonation model (pink), the nickel-surface model (orange), and the collision model (blue).

SN 2018oh (Li et al. 2018). The generic off-center ${ }^{56} \mathrm{Ni}$ model also has a color that is redder than SN $2018 \mathrm{oh}$ by $\sim 0.1 \mathrm{mag}$. On the other hand, the companion-interaction model with a companion at $\approx 2 \times 10^{12} \mathrm{~cm}$ generally matches both the early rise and the color evolution of SN 2018oh.

Based on the color evolution of SN 2018oh, we slightly favor the companion-interaction model over other models that can also reproduce the early flux excess. A direct prediction of this model is the presence of hydrogen and/or helium-rich material stripped from the companion star at the nebular phases (Pan et al. 2012; Liu et al. 2013). To this end, detections of H or He features at late-time spectra of SN 2018oh is crucial to confirm this model.

We note that, while SN 2018oh has an exquisite K2 light curve, we lack the detailed color information to conclusively decide between models, particularly at bluer wavelengths. Additionally, there are no spectra of SN 2018oh during the flux excess, which would have provided key information for 
understanding how spectral features affect the color evolution. We do not see an abrupt flux excess on the first rise component, but rather a linear rise similar to other $\mathrm{SNe}$ Ia with sufficiently early, high-cadence photometry that also have two-component rising light curves (e.g., SN 2012fr, which arguably shows no signs of interaction; Contreras et al. 2018); this argues against the interaction model. A linear rise may result from a particular interaction model (i.e., specific viewing angle, radius, and/or separation); however, an exploration of how different parameter combinations affect the detailed rise behavior is beyond the scope of this Letter.

However, another interpretation of the flux excess is possible. Given the growing sample of SNe Ia that show two-component early light curves, with different slopes and durations, the distinct early light curve evolution of SN 2018oh, compared to the total SN Ia population, could not be due to some external heating source, but rather a reflection of varying $\mathrm{SNe}$ Ia properties, such as the density profile of the ejecta, different composition/metallicity of the progenitor star, asymmetries during the explosion etch (see Stritzinger et al. 2018, for a relevant discussion). Modern transient surveys, such as ASAS-SN and the Zwicky Transient Facility, and future powerful surveys, such as Large Synoptic Survey Telescope, will discover very young $\mathrm{SNe}$ Ia, and with rapid follow-up, the early light curve SN sample will increase, allowing us to investigate this possibility.

\section{Discussion and Conclusions}

In this Letter, we analyze the early photometric observations of SN 2018oh, a normal SN Ia that occurred within the Kepler Campaign 16 field. The SN was observed with unprecedented cadence by the K2 SCE with complementary early PanSTARRS1 and DECam imaging. The combination of an extremely early detection and unprecedented continuous coverage with Kepler makes SN 2018oh a spectacular reference object for early SN Ia studies, providing invaluable insights on the explosion physics and the progenitor system.

In the SN 2018oh K2 and $i$-band light curves, we detect a distinct flux component in the first few days after explosion relative to other well-observed SNe Ia (e.g., SN 2011fe) and the $t^{2}$ luminosity rise seen later in the evolution of SN 2018oh. This flux excess lasts approximately 5 days, after which SN 2018oh appears to evolve in a fashion consistent with typical SNe Ia.

Our work provides new insights on the early-time evolution of $\mathrm{SNe}$ Ia, for which we find the following.

1. The early K2 light curve shows a distinct two-component rise evolution. Initially, the $\mathrm{SN}$ rises quickly, with a steep linear gradient, in flux. This flux subsides after about 5 days, when a $L \propto t^{2}$ rise dominates the $\mathrm{SN}$ evolution.

2. Using the $t^{2}$ component of the $\mathrm{K} 2$ light curve, we constrain $t_{0}$ to be $-18.19 \pm 0.09$ days before $\mathrm{K} 2$ peak brightness. This time is consistent with the onset of the flux excess, indicating that both components began at the same time.

3. Assuming that the $t^{2}$ component exists while the other component is bright, we find that the early flux excess peaks 2.14 days after $t_{0}$, has an FWHM of 3.12 days, a blackbody temperature of $17,500 \mathrm{~K}$, a peak luminosity of $4.3 \times 10^{37} \mathrm{erg} \mathrm{s}^{-1}$, and a total emitted energy of $1.3 \times$ $10^{43} \mathrm{erg}$.
4. We observed SN 2018oh with Pan-STARRS1 and DECam only 4.1 and 20.6 hours after the first K2 detection, respectively, providing some of the earliest colors of a SN Ia ever. The flux excess is confirmed in our ground-based $i$ light curve.

5. The SN 2018oh early photometric evolution is relatively similar to SN 2017cbv, another SN Ia with a prominent two-component rising light curve. However, SN 2018oh shows bluer $\mathrm{K} 2-i$ colors than SN 2017cbv. This is especially true during the epoch of the initial flux excess. Around peak brightness, SN 2018oh is similar to both SNe 2011fe and 2017cbv.

6. The early flux excess can potentially be explained by additional heating at the epoch in question. We investigate three possible sources.

(a) The interaction with a non-degenerate companion at $a=2 \times 10^{12} \mathrm{~cm}$, with an $M \approx 1-6 M_{\odot}$ Roche-lobefilling star.

(b) The presence of a $0.05 M_{\odot}$ helium shell on the surface of $0.98 M_{\odot} \mathrm{C} / \mathrm{O} \mathrm{WD}$, and a subsequent subChandrasekhar-mass explosion.

(c) An off-center ${ }^{56} \mathrm{Ni}$ distribution of $0.03 M_{\odot}$.

All of these models can, generally, reproduce the early shape of the K2 light curve. We slightly favor the interaction scenario, due to the blue colors at the epoch of the flux excess. However, another interpretation of the flux excess considers an intrinsic variation of early-time behavior, due to varying $\mathrm{SNe}$ Ia properties, with no external heating source required.

While an SD origin for (at least a sizable fraction of) SNe Ia has been previously proposed (Sternberg et al. 2011; Foley et al. 2012b; Maguire et al. 2013), its validity has been questioned. Most SNe that have some observational evidence for the presence of a non-degenerate companion are either peculiar (e.g., SNe Ia-CSM; Dilday et al. 2012; Silverman et al. 2013; Fox et al. 2015) or have contradictory observations (Sand et al. 2018; Shappee et al. 2018a). The general progenitor picture that has emerged over the last decade is that most SNe Ia have a DD origin (Maoz et al. 2014).

Excluding the early-time flux excess, SN 2018oh shows no signs of photometric and spectroscopic peculiarities. Therefore, SN 2018oh represents a normal SN Ia with a potential SD origin, challenging the idea that all normal SNe Ia have DD progenitors. Additional SNe Ia observed at high cadence during the first few days after explosion are needed to determine the fraction of $\mathrm{SNe}$ Ia with SD progenitors. At the same time, these observations will grow the early light curve SN Ia sample, and investigate correlations of the light curve evolution with various SNe Ia properties.

The K2 SCE has finished and the data are currently collected and analyzed. With some luck, we will soon have additional K2-observed SNe Ia with data similar in quality to that of SN 2018oh.

We will continue to monitor SN 2018oh. Late-time observations, after the SN has become optically thin, will be a direct test of our proposed models.

We thank the anonymous referee for helpful comments that improved the clarity and presentation of this Letter.

This Letter includes data collected by the K2 mission. Funding for the $\mathrm{K} 2$ mission is provided by the NASA Science Mission directorate. 
KEGS is supported in part by NASA K2 cycle 4 and 5 grants NNX17AI64G and 80NSSC18K0302, respectively. A.R. and his groups is supported in part by $H S T$ grants GO-12577 and HST AR-12851.

Pan-STARRS (PS1) is supported in part by the National Aeronautics and Space Administration under grants NNX12AT65G and NNX14AM74G. The Pan-STARRS1 Surveys (PS1) and the PS1 public science archive have been made possible through contributions by the Institute for Astronomy, the University of Hawaii, the Pan-STARRS Project Office, the Max Planck Society and its participating institutes, the Max Planck Institute for Astronomy, Heidelberg and the Max Planck Institute for Extraterrestrial Physics, Garching, The Johns Hopkins University, Durham University, the University of Edinburgh, the Queen's University Belfast, the Harvard-Smithsonian Center for Astrophysics, the Las Cumbres Observatory Global Telescope Network Incorporated, the National Central University of Taiwan, the Space Telescope Science Institute, the National Aeronautics and Space Administration under grant No. NNX08AR22G issued through the Planetary Science Division of the NASA Science Mission Directorate, the National Science Foundation grant No. AST1238877, the University of Maryland, Eotvos Lorand University (ELTE), the Los Alamos National Laboratory, and the Gordon and Betty Moore Foundation.

This project used data obtained with the Dark Energy Camera (DECam), which was constructed by the Dark Energy Survey (DES) collaboration. Funding for the DES Projects has been provided by the DOE and NSF (USA), MISE (Spain), STFC (UK), HEFCE (UK), NCSA (UIUC), KICP (U. Chicago), CCAPP (Ohio State), MIFPA (Texas A\&M), CNPQ, FAPERJ, FINEP (Brazil), MINECO (Spain), DFG (Germany) and the collaborating institutions in the Dark Energy Survey, which are Argonne Lab, UC Santa Cruz, University of Cambridge, CIEMAT-Madrid, University of Chicago, University College London, DES-Brazil Consortium, University of Edinburgh, ETH Zürich, Fermilab, University of Illinois, ICE (IEEC-CSIC), IFAE Barcelona, Lawrence Berkeley Lab, LMU München and the associated Excellence Cluster Universe, University of Michigan, NOAO, University of Nottingham, Ohio State University, OzDES Membership Consortium, University of Pennsylvania, University of Portsmouth, SLAC National Lab, Stanford University, University of Sussex, and Texas A\&M University.

Based on observations at Cerro Tololo Inter-American Observatory, National Optical Astronomy Observatory (NOAO 2017B-0279; PI: A. Rest, NOAO 2017B-0285; PI: A. Rest), which is operated by the Association of Universities for Research in Astronomy (AURA) under a cooperative agreement with the National Science Foundation.

The UCSC group is supported in part by NASA grants NNG17PX03C and 80NSSC18K0303, NSF grants AST1518052 and AST-1815935, the Gordon \& Betty Moore Foundation, the Heising-Simons Foundation, and by fellowships from the Alfred P. Sloan Foundation and the David and Lucile Packard Foundation to R.J.F.

We thank Chris Burns for providing crucial updates to SNooPy, relevant to the analysis of this Letter.
S.J.S. acknowledges funding from STFC grants ST/ P000312/1 and ST/N002520/1.

This Letter makes use of observations from Las Cumbres Observatory. D.A.H., C.M., and G.H. are supported by the US National Science Foundation grant 1313484. Support for I.A. was provided by NASA through the Einstein Fellowship Program, grant PF6-170148.

J.V. and his group at Konkoly Observatory is supported by the project "Transient Astrophysical Objects" GINOP 2.3.2-152016-00033 of the National Research, Development and Innovation Office (NKFIH), Hungary, funded by the European Union.

This project has been supported by the Lendület Program of the Hungarian Academy of Sciences, projects No. LP2018-7/ 2018 and LP2012-31, and the NKFIH grant K-115709.

Z.s.B. acknowledges the support provided from the National Research, Development and Innovation Fund of Hungary, financed under the PD 17 funding scheme, project No. PD123910.

Research by D.J.S. is supported by NSF grants AST1821967, 1821987, 1813466, and 1813708. N.S. and J.E.A. received support from NSF grant AST-1515559.

We acknowledge the support of the staff of the Lijiang $2.4 \mathrm{~m}$ and Xinglong $2.16 \mathrm{~m}$ telescope. Funding for the LJT has been provided by Chinese Academy of Sciences and the People's Government of Yunnan Province. The LJT is jointly operated and administrated by Yunnan Observatories and Center for Astronomical Mega-Science, CAS. This Letter is supported by the National Natural Science Foundation of China (NSFC grants 11178003, 11325313, and 11633002), and the National Program on Key Research and Development Project (grant No. 2016YFA0400803). J.J.Z. is supported by the National Science Foundation of China (NSFC, grants 11403096, 11773067), the Youth Innovation Promotion Association of the CAS, the Western Light Youth Project, and the Key Research Program of the CAS (grant No. KJZD-EW-M06). T.M.Z. is supported by the NSFC (grants 11203034). This Letter was also partially Supported by the Open Project Program of the Key Laboratory of Optical Astronomy, National Astronomical Observatories, Chinese Academy of Sciences.

E.B. and J.D. acknowledge partial support from NASA grant NNX16AB5G.

C.P.G. acknowledges support from EU/FP7-ERC grant No. [615929].

Parts of this research were supported by the Australian Research Council Centre of Excellence for All Sky Astrophysics in 3 Dimensions (ASTRO 3D), through project number CE170100013.

Support for this Letter was provided by NASA through Hubble Fellowship grant \#HST-HF2-51357.001-A, awarded by the Space Telescope Science Institute, which is operated by the Association of Universities for Research in Astronomy, Incorporated, under NASA contract NAS5-26555, as well as NASA K2 Cycle 4 Grant NNX17AE92G.

\section{Appendix Photometry Tables}


Table 1

SN 2018oh Ground-based Photometry

\begin{tabular}{|c|c|c|c|c|c|}
\hline $\begin{array}{l}\text { UT Date } \\
\text { (YYYY mm dd) }\end{array}$ & $\begin{array}{l}\text { MJD } \\
\text { (Days) }\end{array}$ & $\begin{array}{c}\text { Phase }^{\mathrm{a}} \\
\text { (Rest-frame Days) }^{\text {(R) }}\end{array}$ & $\begin{array}{l}\text { Time from Detection }{ }^{\mathrm{b}} \\
\text { (Rest-frame Days) }\end{array}$ & Filter & $\begin{array}{l}\text { Value } \\
\text { (Mag) }\end{array}$ \\
\hline 2018 Jan 26.24 & 58144.24 & -18.26 & -0.15 & $\bar{i}$ & $23.155^{\mathrm{c}}$ \\
\hline 2018 Jan 26.29 & 58144.29 & -18.21 & -0.10 & $g$ & $23.322^{\mathrm{c}}$ \\
\hline 2018 Jan 26.56 & 58144.56 & -17.94 & 0.17 & $g_{\mathrm{P} 1}$ & $20.852 \pm 0.224$ \\
\hline 2018 Jan 26.57 & 58144.57 & -17.93 & 0.18 & $i_{\mathrm{P} 1}$ & $21.022 \pm 0.268$ \\
\hline 2018 Jan 27.25 & 58145.25 & -17.26 & 0.85 & $\mathrm{i}$ & $19.039 \pm 0.013$ \\
\hline 2018 Jan 27.30 & 58145.30 & -17.21 & 0.90 & $\mathrm{i}$ & $18.957 \pm 0.014$ \\
\hline 2018 Feb 03.33 & 58152.33 & -10.26 & 7.85 & $i_{\mathrm{P} 1}$ & $15.670 \pm 0.004$ \\
\hline 2018 Feb 04.33 & 58153.33 & -9.27 & 8.84 & $i_{\mathrm{P} 1}$ & $15.445 \pm 0.004$ \\
\hline 2018 Feb 04.49 & 58153.49 & -9.11 & 9.00 & $i_{\mathrm{P} 1}$ & $15.388 \pm 0.003$ \\
\hline 2018 Feb 05.40 & 58154.40 & -8.21 & 9.90 & $i_{\mathrm{P} 1}$ & $15.264 \pm 0.010$ \\
\hline 2018 Feb 08.37 & 58157.37 & -5.27 & 12.84 & $g_{\mathrm{P} 1}$ & $14.483 \pm 0.002$ \\
\hline 2018 Feb 09.47 & 58158.47 & -4.18 & 13.93 & $g_{\mathrm{P} 1}$ & $14.359 \pm 0.002$ \\
\hline 2018 Feb 09.48 & 58158.48 & -4.17 & 13.94 & $i_{\mathrm{P} 1}$ & $14.926 \pm 0.003$ \\
\hline 2018 Feb 10.48 & 58159.48 & -3.18 & 14.93 & $g_{\mathrm{P} 1}$ & $14.307 \pm 0.002$ \\
\hline 2018 Feb 11.35 & 58160.35 & -2.32 & 15.79 & $g_{\mathrm{P} 1}$ & $14.285 \pm 0.002$ \\
\hline 2018 Feb 11.35 & 58160.35 & -2.32 & 15.79 & $i_{\mathrm{P} 1}$ & $14.961 \pm 0.003$ \\
\hline 2018 Feb 11.49 & 58160.49 & -2.19 & 15.92 & $g_{\mathrm{P} 1}$ & $14.261 \pm 0.002$ \\
\hline 2018 Feb 12.33 & 58161.33 & -1.36 & 16.76 & $g_{\mathrm{P} 1}$ & $14.241 \pm 0.002$ \\
\hline 2018 Feb 13.56 & 58162.56 & -0.14 & 17.97 & $g_{\mathrm{P} 1}$ & $14.253 \pm 0.002$ \\
\hline 2018 Feb 13.57 & 58162.57 & -0.13 & 17.98 & $i_{\mathrm{P} 1}$ & $15.002 \pm 0.003$ \\
\hline 2018 Feb 14.53 & 58163.53 & 0.82 & 18.93 & $g_{\mathrm{P} 1}$ & $14.225 \pm 0.002$ \\
\hline 2018 Feb 20.34 & 58169.34 & 6.57 & 24.68 & $g_{\mathrm{P} 1}$ & $14.418 \pm 0.002$ \\
\hline 2018 Feb 21.49 & 58170.49 & 7.71 & 25.82 & $g_{\mathrm{P} 1}$ & $14.472 \pm 0.002$ \\
\hline 2018 Mar 07.28 & 58184.28 & 21.35 & 39.46 & $g_{\mathrm{P} 1}$ & $15.465 \pm 0.005$ \\
\hline 2018 Mar 07.29 & 58184.29 & 21.36 & 39.47 & $i_{\mathrm{P} 1}$ & $15.769 \pm 0.006$ \\
\hline 2018 Mar 08.25 & 58185.25 & 22.30 & 40.42 & $g_{\mathrm{P} 1}$ & $15.543 \pm 0.004$ \\
\hline 2018 Mar 08.41 & 58185.41 & 22.46 & 40.57 & $g_{\mathrm{P} 1}$ & $15.514 \pm 0.003$ \\
\hline 2018 Mar 18.29 & 58195.29 & 32.24 & 50.35 & $i_{\mathrm{P} 1}$ & $15.635 \pm 0.006$ \\
\hline 2018 Mar 18.29 & 58195.29 & 32.24 & 50.35 & $g_{\mathrm{P} 1}$ & $16.306 \pm 0.009$ \\
\hline
\end{tabular}

Notes.

${ }^{\text {a }}$ Relative to $\mathrm{MJD}_{B}^{\text {peak }}=58162.7$.

${ }^{\mathrm{b}}$ Relative to $\mathrm{MJD}_{\text {detection }}^{K 2}=58144.39$.

c $3 \sigma$ upper limit.

Table 2

SN 2018oh K2 Photometry

\begin{tabular}{|c|c|c|c|c|}
\hline $\begin{array}{l}\text { UT Date } \\
\text { (YYYY mm dd) }\end{array}$ & $\begin{array}{l}\text { MJD } \\
\text { (Days) }\end{array}$ & $\begin{array}{c}\text { Phase }^{\mathrm{a}} \\
\text { (Rest-frame Days) }\end{array}$ & $\begin{array}{l}\text { Time from Detection }{ }^{\mathrm{b}} \\
\text { (Rest-frame Days) }\end{array}$ & $\begin{array}{l}\text { Value } \\
\text { (Mag) }\end{array}$ \\
\hline 2017 Dec 07.99 & 58094.99 & -66.85 & -48.86 & $20.743^{c}$ \\
\hline 2017 Dec 08.01 & 58095.01 & -66.83 & -48.84 & $20.744^{c}$ \\
\hline 2017 Dec 08.05 & 58095.05 & -66.79 & -48.80 & $20.745^{\mathrm{c}}$ \\
\hline 2017 Dec 08.07 & 58095.07 & -66.77 & -48.78 & $20.745^{\mathrm{c}}$ \\
\hline 2017 Dec 08.09 & 58095.09 & -66.75 & -48.76 & $20.745^{\mathrm{c}}$ \\
\hline 2017 Dec 08.11 & 58095.11 & -66.73 & -48.74 & $20.745^{\mathrm{c}}$ \\
\hline
\end{tabular}

Notes. The complete K2 light curve is available in the electronic edition.

${ }^{\text {a }}$ Relative to $\mathrm{MJD}_{K 2}^{\text {peak }}=58162.1$.

${ }^{\mathrm{b}}$ Relative to $\mathrm{MJD}_{\text {detection }}^{K 2}=58144.39$.

${ }^{\mathrm{c}} 3 \sigma$ upper limit.

(This table is available in its entirety in machine-readable form.)

\section{ORCID iDs}

A. L. Piro (1) https://orcid.org/0000-0001-6806-0673

D. O. Jones (1) https://orcid.org/0000-0002-6230-0151

A. Villar (1) https://orcid.org/0000-0002-5814-4061

G. Narayan (1) https://orcid.org/0000-0001-6022-0484

D. A. Coulter 10 https://orcid.org/0000-0003-4263-2228

C. D. Kilpatrick (1) https://orcid.org/0000-0002-5740-7747
O. D. Fox (1) https://orcid.org/0000-0003-2238-1572

S. W. Jha (1) https://orcid.org/0000-0001-8738-6011

P. E. Nugent (1) https://orcid.org/0000-0002-3389-0586

G. Barentsen (ㄷ) https://orcid.org/0000-0002-3306-3484

J. Dotson (i) https://orcid.org/0000-0003-4206-5649

M. Gully-Santiago (1) https://orcid.org/0000-0002-4020-3457

C. Hedges (i) https://orcid.org/0000-0002-3385-8391 
A. M. Cody (ib https://orcid.org/0000-0002-3656-6706

T. Barclay (ib https://orcid.org/0000-0001-7139-2724

S. Howell i https://orcid.org/0000-0002-2532-2853

P. Garnavich (1) https://orcid.org/0000-0003-4069-2817

B. E. Tucker (1) https://orcid.org/0000-0002-4283-5159

E. Shaya (i) https://orcid.org/0000-0002-3234-8699

S. Margheim (10) https://orcid.org/0000-0001-8205-9441

K. C. Chambers (10 https://orcid.org/0000-0001-6965-7789

H. A. Flewelling (10) https://orcid.org/0000-0002-1050-4056

M. E. Huber (1) https://orcid.org/0000-0003-1059-9603

S. J. Smartt (i) https://orcid.org/0000-0002-8229-1731

J. A. Muñoz iㅏ https://orcid.org/0000-0001-9833-2959

J. Shields (1) https://orcid.org/0000-0002-1560-5286

X. Wang (1) https://orcid.org/0000-0002-7334-2357

J. Zhang (i) https://orcid.org/0000-0002-8296-2590

E. Baron (1) https://orcid.org/0000-0001-5393-1608

G. Hosseinzadeh (1) https://orcid.org/0000-0002-0832-2974

I. Arcavi (1) https://orcid.org/0000-0001-7090-4898

S. Valenti (1) https://orcid.org/0000-0001-8818-0795

J. L. Tonry (i) https://orcid.org/0000-0003-2858-9657

A. N. Heinze (1) https://orcid.org/0000-0003-3313-4921

B. Stalder (1) https://orcid.org/0000-0003-0973-4900

J. Vinkó (1) https://orcid.org/0000-0001-8764-7832

K. Sárneczky (1) https://orcid.org/0000-0003-0926-3950

A. Pál (i) https://orcid.org/0000-0001-5449-2467

Zs. Bognár (ib https://orcid.org/0000-0002-8493-9781

O. Hanyecz (1) https://orcid.org/0000-0002-9415-5219

R. Szabó (1) https://orcid.org/0000-0002-3258-1909

R. Szakáts (i) https://orcid.org/0000-0002-1698-605X

S. C. Williams (1) https://orcid.org/0000-0002-4237-3994

C. Frohmaier (1) https://orcid.org/0000-0001-9553-4723

L. Galbany (i) https://orcid.org/0000-0002-1296-6887

C. P. Gutiérrez (1) https://orcid.org/0000-0002-7252-4351

I. Hook (1) https://orcid.org/0000-0002-2960-978X

C. Inserra $\mathbb{1}$ https://orcid.org/0000-0002-3968-4409

M. Smith ๑ https://orcid.org/0000-0002-3321-1432

D. J. Sand (i) https://orcid.org/0000-0003-4102-380X

\section{References}

Arnett, W. D. 1982, ApJ, 253, 785

Barentsen, G., \& Cardoso, J. V. D. M. 2018, Kadenza: Kepler/K2 Raw

Cadence Data Reader, Astrophysics Source Code Library, ascl:1803.005

Betoule, M., Kessler, R., Guy, J., et al. 2014, A\&A, 568, A22

Bianco, F. B., Howell, D. A., Sullivan, M., et al. 2011, ApJ, 741, 20

Bloom, J. S., Kasen, D., Shen, K. J., et al. 2012, ApJL, 744, L17

Brown, J. S., Stanek, K. Z., Vallely, P., et al. 2018, ATel, 11253, 1

Brown, P. J., Dawson, K. S., Harris, D. W., et al. 2012, ApJ, 749, 18

Burns, C. R., Stritzinger, M., Phillips, M. M., et al. 2011, AJ, 141, 19

Burns, C. R., Stritzinger, M., Phillips, M. M., et al. 2015, SNooPy: TypeIa Supernovae Analysis Tools, Astrophysics Source Code Library, ascl: 1505.023

Cao, Y., Kulkarni, S. R., Gal-Yam, A., et al. 2016, ApJ, 832, 86

Chambers, K. C., Magnier, E. A., Metcalfe, N., et al. 2016, arXiv: 1612.05560

Colgate, S. A., \& McKee, C. 1969, ApJ, 157, 623

Contreras, C., Phillips, M. M., Burns, C. R., et al. 2018, ApJ, 859, 24

Cornect, R., Brimacombe, J., Stone, G., et al. 2018, ATel, 11259, 1

Dilday, B., Howell, D. A., Cenko, S. B., et al. 2012, Sci, 337, 942

Eggleton, P. P. 1983, ApJ, 268, 368

Firth, R. E., Sullivan, M., Gal-Yam, A., et al. 2015, MNRAS, 446, 3895

Fitzpatrick, E. L. 1999, PASP, 111, 63

Flaugher, B., Diehl, H. T., Honscheid, K., et al. 2015, AJ, 150, 150

Foley, R. J., Challis, P. J., Filippenko, A. V., et al. 2012a, ApJ, 744, 38

Foley, R. J., Simon, J. D., Burns, C. R., et al. 2012b, ApJ, 752, 101

Foreman-Mackey, D., Hogg, D. W., Lang, D., \& Goodman, J. 2013, PASP, 125,306

Fox, O. D., Silverman, J. M., Filippenko, A. V., et al. 2015, MNRAS, 447, 772
Ganeshalingam, M., Li, W., \& Filippenko, A. V. 2011, MNRAS, 416, 2607

Ganeshalingam, M., Li, W., Filippenko, A. V., et al. 2012, ApJ, 751, 142

Garnavich, P. M., Tucker, B. E., Rest, A., et al. 2016, ApJ, 820, 23

González-Gaitán, S., Conley, A., Bianco, F. B., et al. 2012, ApJ, 745, 44

Goobar, A., Johansson, J., Amanullah, R., et al. 2014, ApJL, 784, L12

Guy, J., Sullivan, M., Conley, A., et al. 2010, A\&A, 523, A7

Haas, M. R., Batalha, N. M., Bryson, S. T., et al. 2010, ApJL, 713, L115

Hayden, B. T., Garnavich, P. M., Kasen, D., et al. 2010a, ApJ, 722, 1691

Hayden, B. T., Garnavich, P. M., Kessler, R., et al. 2010b, ApJ, 712, 350

Hillebrandt, W., Kromer, M., Röpke, F. K., \& Ruiter, A. J. 2013, FrPhy, 8, 116

Hillebrandt, W., \& Niemeyer, J. C. 2000, ARA\&A, 38, 191

Honscheid, K., DePoy, D. L. \& for the DES Collaboration 2008, arXiv: 0810.3600

Hosseinzadeh, G., Sand, D. J., Valenti, S., et al. 2017, ApJL, 845, L11

Hoyle, F., \& Fowler, W. A. 1960, ApJ, 132, 565

Hsiao, E. Y., Conley, A., Howell, D. A., et al. 2007, ApJ, 663, 1187

Iben, I., Jr., \& Tutukov, A. V. 1984, ApJS, 54, 335

Jiang, J.-A., Doi, M., Maeda, K., et al. 2017, Natur, 550, 80

Jones, D. O., Scolnic, D. M., Riess, A. G., et al. 2018, ApJ, 857, 51

Kasen, D. 2010, ApJ, 708, 1025

Kasen, D., Röpke, F. K., \& Woosley, S. E. 2009, Natur, 460, 869

Kelly, P. L., Fox, O. D., Filippenko, A. V., et al. 2014, ApJ, 790, 3

Kelly, P. L., Hicken, M., Burke, D. L., Mandel, K. S., \& Kirshner, R. P. 2010, ApJ, 715, 743

Kerzendorf, W. E., Childress, M., Scharwächter, J., Do, T., \& Schmidt, B. P. 2014, ApJ, 782, 27

Kerzendorf, W. E., Schmidt, B. P., Laird, J. B., Podsiadlowski, P., \& Bessell, M. S. 2012, ApJ, 759, 7

Kessler, R., Bernstein, J. P., Cinabro, D., et al. 2009, PASP, 121, 1028

Kilpatrick, C. D., Foley, R. J., Drout, M. R., et al. 2018, MNRAS, 473 4805

Kromer, M., Fremling, C., Pakmor, R., et al. 2016, MNRAS, 459, 4428

Lampeitl, H., Smith, M., Nichol, R. C., et al. 2010, ApJ, 722, 566

Leadbeater, R. 2018, TNSCR, 2018, 159

Li, W., Bloom, J. S., Podsiadlowski, P., et al. 2011, Natur, 480, 348

Liu, Z.-W., Pakmor, R., Seitenzahl, I. R., et al. 2013, ApJ, 774, 37

Magnier, E. A., Chambers, K. C., Flewelling, H. A., et al. 2016, arXiv:1612. 05240

Maguire, K., Sullivan, M., Patat, F., et al. 2013, MNRAS, 436, 222

Maoz, D., Mannucci, F., \& Nelemans, G. 2014, ARA\&A, 52, 107

Marion, G. H., Brown, P. J., Vinkó, J., et al. 2016, ApJ, 820, 92

Martínez-Rodríguez, H., Piro, A. L., Schwab, J., \& Badenes, C. 2016, ApJ, 825,57

McCully, C., Jha, S. W., Foley, R. J., et al. 2014, Natur, 512, 54

Miller, A. A., Cao, Y., Piro, A. L., et al. 2018, ApJ, 852, 100

Noebauer, U. M., Kromer, M., Taubenberger, S., et al. 2017, MNRAS, 472, 2787

Nugent, P. E., Sullivan, M., Cenko, S. B., et al. 2011, Natur, 480, 344 Olling, R. P., Mushotzky, R., Shaya, E. J., et al. 2015, Natur, 521, 332

Pakmor, R., Kromer, M., Taubenberger, S., et al. 2012, ApJL, 747, L10

Pan, K.-C., Ricker, P. M., \& Taam, R. E. 2012, ApJ, 750, 151

Pereira, R., Thomas, R. C., Aldering, G., et al. 2013, A\&A, 554, A27

Perlmutter, S., Aldering, G., Goldhaber, G., et al. 1999, ApJ, 517, 565

Perlmutter, S., Gabi, S., Goldhaber, G., et al. 1997, ApJ, 483, 565

Piro, A. L., Chang, P., \& Weinberg, N. N. 2010, ApJ, 708, 598

Piro, A. L., \& Morozova, V. S. 2016, ApJ, 826, 96

Piro, A. L., \& Nakar, E. 2013, ApJ, 769, 67

Rest, A. 2018, ATel, 11345, 1

Rest, A., Garnavich, P. M., Khatami, D., et al. 2018a, NatAs, 2, 307

Rest, A., Scolnic, D., Foley, R. J., et al. 2014, ApJ, 795, 44

Rest, A., Strampelli, G. M., Zenteno, A., et al. 2018b, ATel, 11344, 1

Rest, A., Stubbs, C., Becker, A. C., et al. 2005, ApJ, 634, 1103

Riess, A. G., Filippenko, A. V., Challis, P., et al. 1998, AJ, 116, 1009

Riess, A. G., Filippenko, A. V., Li, W., et al. 1999, AJ, 118, 2675

Sand, D. J., Graham, M. L., Botyánszki, J., et al. 2018, arXiv:1804.03666

Schaefer, B. E., \& Pagnotta, A. 2012, Natur, 481, 164

Schechter, P. L., Mateo, M., \& Saha, A. 1993, PASP, 105, 1342

Schlafly, E. F., \& Finkbeiner, D. P. 2011, ApJ, 737, 103

Scolnic, D. M., Jones, D. O., Rest, A., et al. 2018, ApJ, 859, 101

Shappee, B. J., Piro, A. L., Holoien, T. W.-S., et al. 2016, ApJ, 826, 144

Shappee, B. J., Holoien, T. W.-S., Drout, M. R., et al. 2018b, ApJ, 870, 13

Shappee, B. J., Piro, A. L., Stanek, K. Z., et al. 2018a, ApJ, 855, 6

Shappee, B. J., Prieto, J. L., Grupe, D., et al. 2014, ApJ, 788, 48

Shaya, E. J., Olling, R., \& Mushotzky, R. 2015, AJ, 150, 188

Silverman, J. M., Nugent, P. E., Gal-Yam, A., et al. 2013, ApJS, 207, 3 
Sim, S. A., Fink, M., Kromer, M., et al. 2012, MNRAS, 420, 3003

Sim, S. A., Seitenzahl, I. R., Kromer, M., et al. 2013, MNRAS, 436, 333

Smith, K. W., Rest, A., Tucker, B. E., et al. 2018, ATel, 11218, 1

Sternberg, A., Gal-Yam, A., Simon, J. D., et al. 2011, Sci, 333, 856

Stritzinger, M. D., Shappee, B. J., Piro, A. L., et al. 2018, arXiv:1807.07576

Sullivan, M., Conley, A., Howell, D. A., et al. 2010, MNRAS, 406, 782

Taylor, E. N., Hopkins, A. M., Baldry, I. K., et al. 2011, MNRAS, 418, 1587
Tucker, B. E. 2011, Ap\&SS, 335, 223

Waters, C. Z., Magnier, E. A., Price, P. A., et al. 2016, arXiv:1612.05245

Whelan, J., \& Iben, I., Jr. 1973, ApJ, 186, 1007

Woosley, S. E., \& Kasen, D. 2011, ApJ, 734, 38

Woosley, S. E., Taam, R. E., \& Weaver, T. A. 1986, ApJ, 301, 601

Woosley, S. E., \& Weaver, T. A. 1994, ApJ, 423, 371

Zhang, J., Xin, Y., Li, W., et al. 2018, ATel, 11267, 1 